\title{
CHANGES IN THE FLORISTIC COMPOSITION AND ECOLOGY OF RUDERAL FLORA OF THE TOWN OF KOSOVSKA MITROVICA, SERBIA FOR A PERIOD OF 20 YEARS
}

\author{
ProdAnOviĆ, D. ${ }^{1 *}$ - KrIVOŠEJ, Z. ${ }^{2}-$ AMIDŽIĆ, L. $^{3}-$ BIBERDŽIĆ, M. ${ }^{1}-$ KRSTIĆ, Z. ${ }^{2}$ \\ ${ }^{1}$ University of Priština, Faculty of Agriculture Lešak \\ Kopaonička Street bb, 38219 Lešak, Serbia (phone: + 3816400727 87) \\ ${ }^{2}$ University of Priština, Faculty of Natural Science \\ Lole Ribara Street, No. 29, 38220 Kosovska Mitrovica, Serbia \\ ${ }^{3}$ University Union Nikola Tesla, Faculty of Ecology and Environmental Protection \\ Cara Dušana street, No. 62-64, 11000 Belgrade, Serbia \\ *Corresponding author \\ e-mail: danijela.prodanovic@gmail.com \\ (Received 23 $3^{\text {rd }}$ May 2017; accepted $2^{\text {nd }}$ Aug 2017)
}

\begin{abstract}
The paper is concerned with the results of the ruderal flora investigation carried out in the vicinity of the town of Kosovska Mitrovica (Serbia) and its surroundings, in different urban and suburban habitats, and is based on the copious floristic researches conducted between 1995 and 1996 and repeated in 2016. The total number of 444 taxa was reported in the course of 2016. Not only was reported the presence of 386 taxa in the same areas between 1995 and 1996, but also 58 new taxa were recorded in recent field explorations. The ruderal flora composition in Kosovska Mitrovica area has changed by $13.06 \%$ in the past 20 years. Detailed taxonomic, ecological, and phyto-geographical analyses were provided for the discovered synanthropic flora. Special attention was paid to the appearance of new invasive species unregistered 20 years ago, but which, due to the more intensive anthropogenic influence, have become more diverse in number and frequency in the investigated areas.
\end{abstract}

Keywords: urban habitats, ruderal flora, ecological analysis, invasive plants, Kosovska Mitrovica

\section{Introduction}

Urbanization has created new ecosystems that harbor specialized flora adapted to anthropogenic alterations (Neto et al., 2015). The ecological approach considers a city/town as an ecosystem. "Ruderal" biocenoses exist in all cities. The term "ruderata" (from Lat. rudus: rubble, ruins) refers to a specific habitat. The definition connects ruderal plants with their habitats: they grow in places (such as rubble) which are strongly disturbed by man, but not cultivated (Sukopp, 2002). The habitats with modified physical and chemical soil properties have lost their resemblance to primary land type and are located in the vicinity of settlements and around all types of urban buildings and infrastructures (Prodanović et al., 2008). Other terms used for the flora in human settlements are synanthropic, synurban and nitrophile flora (PavlovićMuratspahić et al., 2010). Anthropogenic factors are of essential importance in the formation, survival, distribution, diversity and dynamics of this type of flora/vegetation (Jarić et al., 2011).

The flora of urban habitats has long been recognized as being rich in species (Stešević and Jovanović, 2008). Urbanization transforms floras through a series of filters that change: (i) habitat availability;(ii) the spatial arrangement of habitats; (iii) the pool of plant species; and (iv) evolutionary selection pressures on populations 
persisting in the urban environment. Habitat transformation and fragmentation are anthropogenic filters present in most ecosystems, while the strong influences of human preference and urban environmental conditions are unique to cities (Williams et al., 2008). The development and importance of this spontaneously developed plant cover are often underestimated in urban ecosystem by constant human efforts to destroy and curb it by giving way to cultivated plants. Ruderal plant species are often considered as invasive weeds; some have medicinal value while others have unknown utility. Regardless of its specific use, the ruderal flora grows rapidly and thus serves as study material for many botanical subareas and provides an opportunity to study the development of vegetation when most plants are annuals. The ecology of ruderal plants also reinforces the importance of these plants because they can vegetate, bloom, and fructify with high efficiency, allowing them to be used for the recovery of degraded areas (Neto et al., 2015).

Ruderal and synanthropic (synurban) floras, very young and dynamic floristic assemblages, as well as the corresponding vegetation units, have recently drawn attention of scientists throughout the world (Jovanović, 1997). The research of synanthropic flora of various urban areas (cities, towns and smaller settlements) has had a long tradition. The interest in urban floras can be attributed to the fact that cities are remarkably ample with species due to a high habitat diversity and abundance of alien species. Outside Europe, urbanization and its consequences have been intensively studied in North America (Celesti-Grapow et al., 2006). The first investigations in the field of urban ecology in Europe focused on single habitat types (old settlements, ruins, gardens, and parks), while comprehensive studies of urban ecosystems were started in the 1970's (Sukopp, 2002). In Serbia, as a part of territory of former Yugoslavia, similarly to other European countries, such studies were conducted in the second half of the $19^{\text {th }}$ century (Šajinović, 1968; Slavnić, 1951, 1960, 1961; Marković, 1964, 1978, 1984; Matvejeva, 1982; Jarić, 2000), while special interest in the urban/ruderal flora and vegetation appeared in works of Jovanović (1997), Jovanović and Mitrović (1998), Stankovic-Kalezić (2007), Stanković-Kalezić et al. (2008; 2009).

The floristic research of ruderal weed was conducted to determine accurate data about the qualitative composition of ruderal flora and the quantitative presence of certain species on ecologically diverse surfaces of urban and suburban areas of the town of Kosovska Mitrovica (Serbia). The purpose of the paper was to identify possible changes in the floristic composition of ruderal flora for a period of 20 years, which might provide data on the succession planting and fully introspect ecology of each individual plant species in the investigated area.

\section{Materials and Methods}

The investigation was carried out in the central area of the town of Kosovska Mitrovica (Serbia) and its surroundings, on various urban and suburban ruderal surfaces within more than 15 kilometers. During the growing season of 1995-1996, field researches were conducted on 28 randomly chosen sampling sites (trodden and nitrified ruderal places, nitrified ruderal non-trodden habitats, hygrophilic ruderal habitats, covered disposal areas). During 2016 the researches were continued in the majority of the previously investigated areas but it was virtually impossible to provide a detailed map of already studied probing areas because they had absolutely changed due to extreme anthropogenic influences (i.e. urbanization). Likewise, as the town had 
extended to new areas, another two probing surfaces were added during floristic investigations in 2016. The majority of collected plant material is kept in the Herbarium at the Institute of Botany and the Botanical Garden of Faculty of Biology, University of Belgrade (BEOU).

The collected plants were determined on the basis of modern floristic literature: The Flora of Serbia: Josifović, ed. 1970-1977; Sarić and Diklić, eds. 1989 and The Flora of Europe: Tutin, ed. 1964-1980. The nomenclature was adjusted to Euro+Med Plantbase (2006) and The Plant List (2013). The life forms of plants were determined by applying Ellenberg and Muller-Dambois method (1967), with Stevanović's supplements (1992) and elaborations based on the conditions in Serbia. Stevanović's (1992a) phytogeographical classification was the starting point for determination and phyto analysis of floral elements. The prevalence was calculated by the number of sites where the target species occurred divided by the total number (30) of sampling sites.

\section{Study area}

The town of Kosovska Mitrovica, with its surroundings, lies between $42^{\circ} 53^{\prime}$ north latitude, $20^{\circ} 52^{\prime}$ east longitude, and 496-510 altitude, and stretches to the farthest northern point of Kosovo Valley (Fig. 1). It is situated in the alluvial area and on the terraces of the Ibar and Sitnica Rivers, with the volcanic cup of Zvečan dominating the city. The largest part of the territory is considered mountaineous. More than half of the city and its surroundings consists of brown shallow soil with poor fertility. It should be emphasized that, influenced by heavy industry, many types of soil have undergone degradation and "enrichment" with various waste products that reduced their fertility. The climate is marked as moderate-continental. Mitrovica is located among Kopaonik and Rogozna mountains, hence the annual precipitation is low (600 $\mathrm{mm}$ on average).

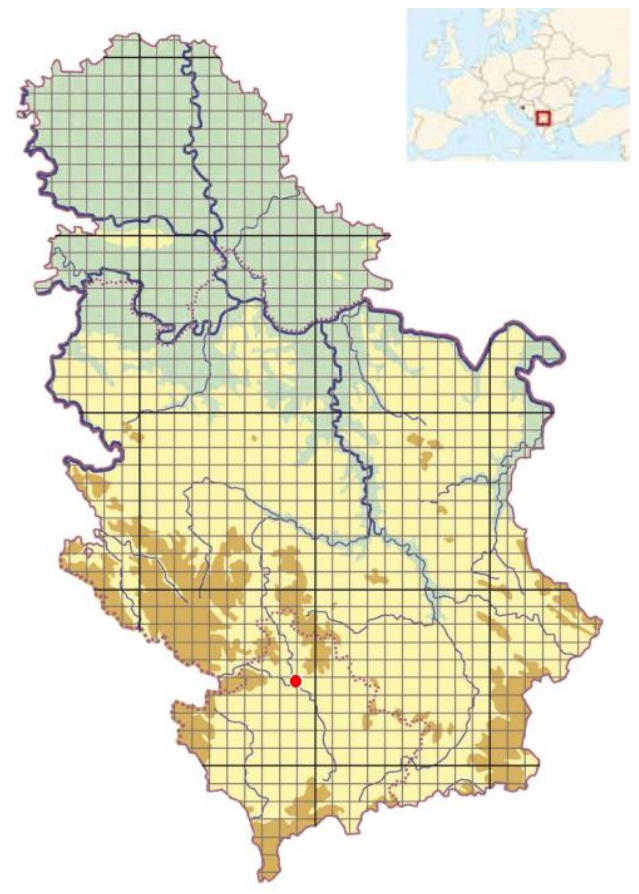

Figure 1. Geographical position of investigated area (town of Kosovska Mitrovica) in Serbia (marked with red point) 


\section{Results and Discussion}

\section{Taxonomic spectrum of the ruderal flora in the town of Kosovska Mitrovica}

Ruderal species are plants commony grown in urban areas and are best adapted to man-altered environments. Floristic researches in different types of ruderal habitats in the city proper of Kosovska Mitrovica and its surroundings, within the range of 15 kilometers, discovered 444 species of vascular plants representing 271 genera and 58 families (Table 1).

The study presents a continuation of the research, whose results were published in 2008 (Prodanović et al., 2008). The presence of 386 taxa was reported in the same areas between 1995 and 1996, and 58 new taxa were recorded in recent field explorations. It is important to emphasize that not all collected species are ruderal, even though they can be traced in various anthropogenic habitats. A certain number of species are found in habitats of primary or secondary forms of vegetation, such as forest, meadows or completely segetal (field) species. These species apperently present either the debris of the original primary communities that were prior to ruderal communities or they appeared afterwards.

Newly recorded taxa include: Allium flavum L., Chaerophyllum bulbosum L., Vinca minor L., Hedera helix L., Asparagus tenuifolius Lam., Ambrosia artemisifolia L., Cota austriaca (Jacq.) Sch. Bip, Symphyotrichum novi-belgii (L.) G.L.Nesom, Symphyotrichum salignum (Willd.) G.L.Nesom, Calendula officinalis L., Echinops sphaerocephalus L., Filago germanica (L.) Huds., Helianthus tuberosus L., Lactuca muralis (L.) Gaertn., Lactuca perennis L., Solidago gigantea Aiton, Tanacetum corymbosum (L.) Sch.Bip., Catalpa bignonioides Walter., Anchusa azurea Mill., Cynoglossum creticum Mill., Nonnea pulla DC., Arabidopsis thaliana (L.) Heynh., Camelina sativa (L.) Crantz., Sisymbrium officinale (L.) Scop., Sisymbrium orientale L., Blitum bonus-henricus (L.) Rchb., Cuscuta europaea L., Echinocystis lobata (Michx.) Torr.\& A. Gray, Carex panicea L., Shoenoplectus lacuster (L.) Palla, Euphorbia taurensis All., Amorpha fruticosa L., Trifolium arvense L., Vicia tenuifolia Roth., Vicia tetrasperma (L.) Schreb., Geranium columbinum L., Leonurus cardiaca L., Malva neglecta Wallr., Maclura pomifera (Raf.) C.K. Schneid., Fraxinus ornus L., Phytolacca americana L., Platanus orientalis L., Arrhenatherum elatius (L.) J. Presl \& C. Presl, Bromus arvensis L., Poa trivialis L., Reseda phyteuma L., Sherardia arvensis L., Populus alba L., Populus nigra L., Salix alba L., Comandra umbellata (L.) Nutt., Acer negundo L., Acer pseudoplatanus L., Acer saccharinum L., Antirrhinum majus L., Veronica praecox All., Veronica serpyllifolia L. and Viola arvensis Murray.

The taxonomic spectrum of the vascular flora in the city area of Kosovska Mitrovica includes 3 classes, 58 families, 271 genera and 444 species and subspecies. Dicotyledones, with 51 families, 231 genera $(49,09 \%)$ and 384 taxa $(86,03 \%)$, are richer in number than Monocotyledones which include 6 families $(1,35 \%), 41$ genera $(9,23 \%)$ and 60 taxa $(13,51 \%)$, whereas horsetail (Equisetinae) is present only with two species of Equisetaceae. According to the researches of Pyšek et al. (2009) the most common families in the urban areas of Europe are: Asteraceae, Poaceae, Rosaceae, Fabaceae and Brassicaceae, which, along with ruderal species, comprise farming weed and invasive species; their number is positively related to the urbanization level (Pyšek, 1998). 
Table 1. Overview of ruderal flora of Kosovska Mitrovica (Serbia) with its life forms, floristic elements and prevalence (\%x100)

\begin{tabular}{|c|c|c|c|}
\hline Family/Species & Life forms & Floristic element & Prevalence \\
\hline \multicolumn{4}{|l|}{ ALLICEAE } \\
\hline Allium flavum L. & v-a Mes-Mac G bulb & apen(s)-ilir-balk-dac-pan & $3,3 \%$ \\
\hline Allium vineale L. & v-a Mes-Meg G bulb & med-subm-pan-atl-ce & $6,6 \%$ \\
\hline \multicolumn{4}{|l|}{ ADOXACEAE } \\
\hline Sambucus ebulus L. & a Alt G rad scap/a H scap & se-med-subm-pont-j.sib-or-tur & $10 \%$ \\
\hline Sambucus nigra L. & fo dec Mi P scap & se-med-subm-pont-j.sib & $10 \%$ \\
\hline \multicolumn{4}{|l|}{ AMARANTHACEAE } \\
\hline Amaranthus albus L. & a Meg T scap & adv (sam-sram) & $6,6 \%$ \\
\hline Amaranthus blitoides S. Watson & a Mes-Meg T rept & $\operatorname{adv}(\mathrm{sam})$ & $10 \%$ \\
\hline Amaranthus retroflexus L. & a Mes-Alt T scap & $\operatorname{adv}(\mathrm{sam})$ & $30 \%$ \\
\hline \multicolumn{4}{|l|}{ APIACEAE } \\
\hline Aegopodium podagraria L. & a Meg-Alt G rhiz scap & se-med-subm-pont-j.c.sib & $13,3 \%$ \\
\hline Anthriscus caucalis M. Bieb. & a Mes-Meg T scap & subevr-az. & $23,3 \%$ \\
\hline $\begin{array}{l}\text { Anthriscus cerefolium (L.) Hoffm. } \\
\text { var. trichospermus Endl. }\end{array}$ & a Meg T scap & i.subm-pan-pont-tur & $26,6 \%$ \\
\hline Anthriscus sylvestris (L.) Hoffm. & a Meg-Alt H scap & se-med-subm-pont-j.sib-i.afr & $23,3 \%$ \\
\hline Bifora radians M.Bieb. & v-a Mes-Meg T scap & c.ev-c.i.med-subm-pont-or-tur & $16,6 \%$ \\
\hline Chaerophyllum bulbosum L. & a Mes-Alt H/T scap bienn & c.ev-med-subm-pont & $16,6 \%$ \\
\hline Conium maculatum L. & a Meg H scap bienn & se-med-subm-pont-j.sr-sib-or-tur-ca-i.j.afr & $23,3 \%$ \\
\hline Daucus carota L. & a Meg H scap/a T scap & se-med-pont-or-tur-i.afr & $36,6 \%$ \\
\hline Eryngium campestre L. & a Mes-Meg H scap & med-subm-pont & $43,3 \%$ \\
\hline Falcaria vulgaris Bernh. & a Mes-Meg T scap & se(z.ev-j.sarm)-pont-or-tur-ca & $23,3 \%$ \\
\hline Foeniculum vulgare Mill. & a Meg-Alt H scap & adv (med-or-tur) & $10 \%$ \\
\hline Heracleum sphondylium L. & a Meg-Alt H scap & se-ev (bor)-med-subm-pont-j.c.sib & $36,6 \%$ \\
\hline Orlaya grandiflora (L.) Hoffm. & a Meg T scap & c.ev-med-subm-pan-z.pont & $30 \%$ \\
\hline Pastinaca sativa L. & a Meg H scap bienn & se-med-pont-j.sib & $30 \%$ \\
\hline Scandix pecten-veneris L. & v Mes-Meg T scap & se-med-subm-or-tur-ca & $43,3 \%$ \\
\hline Torilis arvensis (Huds.) Link & a Meg T scap & kosm (ev-med) & $16,6 \%$ \\
\hline \multicolumn{4}{|l|}{ APOCYNACEAE } \\
\hline Vinca minor L. & Mi-Mes Ch suff rept & adv (se, kult) & $6,6 \%$ \\
\hline \multicolumn{4}{|l|}{ ARALIACEAE } \\
\hline Hedera helix L. & semp S lig & atl-se-med-subm (ev) & $13,3 \%$ \\
\hline
\end{tabular}




\begin{tabular}{|c|c|c|c|}
\hline \multicolumn{4}{|l|}{ ARISTOLOCHIACEAE } \\
\hline Aristolochia clematitis L. & a Mes-Meg G rad scap & subm-pont & $23,3 \%$ \\
\hline \multicolumn{4}{|l|}{ ASPARAGACEAE } \\
\hline Asparagus tenuifolius Lam. & a Meg G rhiz caesp & c.subm-pan-z.pont & $3,3 \%$ \\
\hline \multicolumn{4}{|l|}{ ASTERACEAE } \\
\hline Achillea millefolium L. & a Meg H scap & evr (bor-submerid) & $60 \%$ \\
\hline Ambrosia artemisifolia L. & Aut Meg T scap & $\operatorname{adv}(\mathrm{sam})$ & $6,6 \%$ \\
\hline Anthemis arvensis L. & a Mes-Meg T scap/a H scapp & med-subm & $26,6 \%$ \\
\hline Cota austriaca (Jacq.) Sch. Bip & a Mes-Meg T scap bienn & pont-pan & $36,6 \%$ \\
\hline Cota tinctoria (L.) J. Gay & a Meg H scap bienn & se-med-subm-or-pont & $19 \%$ \\
\hline Arctium lappa L. & aut Meg-Alt H scap bienn & evr (temp-submerid) & $43,3 \%$ \\
\hline Artemisia absinthium L. & Meg Ch suff caesp & evr (subbor-merid) & $30 \%$ \\
\hline Artemisia scoparia Waldst.\& Kit. & aut Meg-Alt H scap bienn & evr ( subbor-submerid) & $36,6 \%$ \\
\hline Artemisia vulgaris L. & aut Meg- Alt H scap & evr-sam (subbor-merid) & $60 \%$ \\
\hline Symphyotrichum novi-belgii (L.) G.L.Nesom & aut Meg-Alt H scap & $\operatorname{adv}(\mathrm{sam})$ & $10 \%$ \\
\hline Symphyotrichum salignum (Willd.) G.L.Nesom & aut Meg-Alt H scap & $\operatorname{adv}(\mathrm{sam})$ & $10 \%$ \\
\hline Bellis perennis L. & a Mes $\mathrm{H}$ ros & se-med-subm & $60 \%$ \\
\hline Bidens tripartitus L. & aut Mes-Alt T scap & evr (subbor-temp) & $6,6 \%$ \\
\hline Calendula officinalis L. & a Mes-Meg T scap & $\operatorname{adv}($ med, kult) & $10 \%$ \\
\hline Carduus acanthoides L. & a Meg-Alt H scap bienn & se-med-subm-pont-j.c.sib-or-tur & $23,3 \%$ \\
\hline Carlina vulgaris L. & a Meg H scap & se-subm-pont-j.sib & $3,3 \%$ \\
\hline Centaurea jacea L. & a Meg-Alt H scap & evr (subbor-submerid) & $40 \%$ \\
\hline Centaurea orientalis L. & a Meg-Alt H scap & pan-z.pont & $36,6 \%$ \\
\hline Centaurea scabiosa L. & a Meg-Alt H scap & se-med-pont-j.c.sib-tur & $36,6 \%$ \\
\hline Centaurea solstitialis L. & a Meg T scap & med-subm-or-pont-j.sib-tur & $50 \%$ \\
\hline $\begin{array}{l}\text { Centaurea stoebe L. } \\
\text { subsp. australis (Pančić ex A.Kern.) Greuter }\end{array}$ & a Meg H scap & pan-z.pont & $53,3 \%$ \\
\hline Chondrilla juncea L. & a Meg-Alt H scap & med-subm-or-pont-j.sib-tur & $10 \%$ \\
\hline Cichorium intybus L. & a-aut Meg-Alt H scap & kosm (evr) & $50 \%$ \\
\hline Cirsium arvense (L.) Scop. & a Meg-Alt G rad scap & evr (subbor-merid) & $46,6 \%$ \\
\hline Cirsium candelabrum Griseb. & a Alt H scap bienn & balk. (end) & $23,3 \%$ \\
\hline Cirsium creticum (Lam.) d'Urv. & a Meg H scap bienn & med-subm & $20 \%$ \\
\hline Cirsium vulgare (Savi) Ten. & a Meg-Alt H scap bienn & evr (subbor-merid) & $16,6 \%$ \\
\hline Crepis biennis L. & a Meg-Alt H scap bienn & se-subm-pont & $10 \%$ \\
\hline Crepis nicaeensis Pers. & a Mes-Meg T scap/a H scap bienn & med-subm-or & $10 \%$ \\
\hline
\end{tabular}




\begin{tabular}{|c|c|c|c|}
\hline Crepis rhoeadifolia M.B. Fiori et Paol. & a Meg-Alt H scap bienn & se-subm-pont & $30 \%$ \\
\hline Crepis sancta (L.) Bornm. & v-a Mes-Meg T ros/H ros & pont & $20 \%$ \\
\hline Crepis setosa Haller & a Mes-Meg T scap & c.i.med-subm-or-z.pont & $20 \%$ \\
\hline Cyanus segetum Hill. & a Mes-Meg T scap & kosm (med) & $36,6 \%$ \\
\hline Echinops sphaerocephalus L. & a Meg-Alt H scap & se-subm-pont-j.sib & $23,3 \%$ \\
\hline Erigeron acer L. & a Mes-Meg T scap & evr-az & $50 \%$ \\
\hline Erigeron canadensis L. & a Meg-Alt T scap & adv (sam) & $53,3 \%$ \\
\hline Eupatorium cannabinum L. & a Meg-Alt H scap & se-med-subm-pont-j.sib & $16,6 \%$ \\
\hline Filago germanica (L.) Huds. & a Mes T scap & se-med-subm-or-pont-j.sib-tur & $10 \%$ \\
\hline Galinsoga parviflora Cav. & a Mes-Meg T scap & $\operatorname{adv}($ jam) & $40 \%$ \\
\hline Helianthus tuberosus L. & a Meg-Alt G bulb & $\operatorname{adv}($ sram) & $10 \%$ \\
\hline Inula britannica L. & a Mes-Meg H scap & evr (temp-merid) & $16,6 \%$ \\
\hline Inula conyzae (Griess.) DC. & a Meg-Alt H scap bienn/a H scap & ev-z.az & $16,6 \%$ \\
\hline Lactuca muralis (L.) Gaertn. & a Mes-Meg H scap & evr (bor-submerid) & $30 \%$ \\
\hline Lactuca perennis L. & a Mes-Me H scap & subm & $30 \%$ \\
\hline Lactuca serriola L. & a Meg-Alt H scap bienn/a T scap & evr (subbor-merid)-i.afr ( boreosubtrop) & $66,6 \%$ \\
\hline Lactuca viminea (L.) J Presl.\& C.Presl. & a Meg-Alt H scap bienn & med-subm-pont-pan-ir (w)-boh-burgd. & $16,6 \%$ \\
\hline Lapsana communis L. & a Meg-Alt T scap & se-med-subm-pont-or-j.sib-ca & $10 \%$ \\
\hline Leontodon crispus DC, ex Nyman & a Mi-Mes H ros & med-i.subm-pont-j.sib & $13,3 \%$ \\
\hline Leucanthemum vulgare (Vaill.) Lam. & v-aut Mes-Meg H scap & evr (bor-merid) & $30 \%$ \\
\hline Matricaria chamomilla L. & a Mi-Mes T scap & kosm (subm) & $56,6 \%$ \\
\hline Onopordum acanthium L. & a Meg-Alt H scap bienn & evr (temp-merid) & $16,6 \%$ \\
\hline Petasites hybridus (L.) G.Gaertn., B.Mey \&Schaerb & a Mes-Meg G rad & evr (bor-submerid) & $16,6 \%$ \\
\hline Picris hieracioides L. & a Meg-Alt H scap bienn/a H scap & evr (temp-merid) & $23,3 \%$ \\
\hline Pilosella bauhinii (Schult) Arv.-Touv. & a Mes H ros rept & c.ev.-sarm-pont-j.sr.sib & $40 \%$ \\
\hline Pilosella officinarum Vaill. & a Mi-Mac H ros & SJEP & $40 \%$ \\
\hline Podospermum laciniatum (L.) DC. & a Mi-Meg T scap bienn/H scap & pont-c.az.-subm & $23,3 \%$ \\
\hline Pulicaria dysenterica (L.) Bernh. & a Mes Meg H scap & se-med-subm-pont-or-tur & $16,6 \%$ \\
\hline $\begin{array}{l}\text { Senecio leucanthemifolium subsp. vernalis ( Waldst.\& Kit.) } \\
\text { Greuter }\end{array}$ & v Mes-Meg T scap & ev-med-subm-or-pont-j.sib & $30 \%$ \\
\hline Senecio vulgaris L. & v-aut Mi-Meg T scap & kosm (evr) & $43,3 \%$ \\
\hline Solidago gigantea Aiton & a Meg-Alt H scap & $\operatorname{adv}(\mathrm{sam})$ & $10 \%$ \\
\hline Solidago virgaurea L. & a Meg-Alt H scap & evr-sam (bor-temp) & $10 \%$ \\
\hline $\begin{array}{l}\text { Sonchus arvensis L. } \\
\text { var. arvensis }\end{array}$ & a Meg-Alt H scap & kosm (evr) & $23,3 \%$ \\
\hline
\end{tabular}




\begin{tabular}{|c|c|c|c|}
\hline $\begin{array}{l}\text { Sonchus arvensis L. } \\
\text { subsp.. uliginosus (M.Bieb.) Nyman }\end{array}$ & a Meg-Alt H scap & kosm (evr) & $8-26,6 \%$ \\
\hline $\begin{array}{l}\text { Sonchus asper (L.) Hill. } \\
\text { subsp. asper }\end{array}$ & a Meg-Alt T scap/a H scap bienn & kosm (med-subm) & $9-30 \%$ \\
\hline $\begin{array}{l}\text { Sonchus asper (L.) Hill. } \\
\text { subsp. glaucescens (Jord.) Ball }\end{array}$ & a Meg-Alt H scap bienn & ev.-z.az & $9-30 \%$ \\
\hline Sonchus oleraceus L. & a Meg-Alt T scap/a H scap bienn & kosm (med-subm) & $33,3 \%$ \\
\hline Tanacetum corymbosum (L.) Sch.Bip. & a Meg-Alt H scap & se-med-subm-pont-j.sib & $10 \%$ \\
\hline Tanacetum vulgare L. & a Meg-Alt H scap & evr (temp-merid) & $23,3 \%$ \\
\hline Taraxacum officinale F.H.Wigg. & v-aut Mes H ros & kosm (evr) & $76,6 \%$ \\
\hline Tragopogon dubius Scop. & a Mes-Meg H scap bienn & se-subm-pont-or & $50 \%$ \\
\hline $\begin{array}{l}\text { Tragopogon dubius Scop. } \\
\text { subsp. major (Jacq.) Vollm. }\end{array}$ & a Mes-Meg H scap bienn & & $50 \%$ \\
\hline Tragopogon pratensis L. & a Meg H scap & subm (ev) pont-j.c.sib-tur & $63,3 \%$ \\
\hline Tripleurospermum inodorum (L.) Sch.Bip & a-aut Meg T scap/ H scap bienn & kosm (med-subm) & $50 \%$ \\
\hline Tussilago farfara L. & v Mi-Mes G rhiz & se-med-subm-pont-j.sib-ca & $23,3 \%$ \\
\hline Xanthium orientale subsp. italicum (Moretti) Greuter & a Meg-Alt T scap & $\operatorname{adv}(\mathrm{sam})$ & $26,6 \%$ \\
\hline Xanthium spinosum L. & a Mes-Meg T scap & kosm (jam) & $33,3 \%$ \\
\hline Xeranthemum annuum L. & a Mes-Meg T scap & med-subm-or-z.pont & $43,3 \%$ \\
\hline \multicolumn{4}{|l|}{ BIGNONIACEAE } \\
\hline Catalpa bignonioides Walter. & v-a Mes P scap & subm & $3,3 \%$ \\
\hline \multicolumn{4}{|l|}{ BORAGINACEAE } \\
\hline Aegonychon purpurocaeruleum (L.) Holub & v Mes-Meg H scap & pont-subm & $20 \%$ \\
\hline Anchusa azurea Mill. & a Mes-Meg H scap & med-subm-pan-pont-or & $16,6 \%$ \\
\hline Anchusa officinalis L. & a Meg H scap bienn/a H scap & se-i.subm-pan-z.pont & $46,6 \%$ \\
\hline Asperugo procumbens L. & v Mes-meg T scap & evr (bor-submerid)-sam (sin.) & $10 \%$ \\
\hline Buglossoides arvensis (L.) I.M.Johnst. & V-a N-Meg T scap & evr (subbor-merid) & $16,6 \%$ \\
\hline Cerinthe minor L. & v-a Mes-Meg H scap bienn/T scap & (ev)-med-subm-z.pont & $23,3 \%$ \\
\hline Cynoglossum creticum Mill. & a Mes-Meg H scap bienn & atl-med-subm-tur & $16,6 \%$ \\
\hline Cynoglossum officinale L. & a Mes-Meg H scap bienn & se-med-subm-pont-sr.sib & $26,6 \%$ \\
\hline Echium italicum L. & a-aut Meg H scao bienn & med-subm & $20 \%$ \\
\hline Echium vulgare L. & a Mes-Alt H scap bienn/a H scap & se-med-subm-pont-j.sib & $30 \%$ \\
\hline Heliotropium europaeum L. & a Mes-Meg T scap & med-subm-pan-z.pont & $10 \%$ \\
\hline Myosotis arvensis (L.) Hill. & a Mes H scap bienn/a T scap & se-med-subm-pan-pont-or & $16,6 \%$ \\
\hline Myosotis sparsiflora Pohl & V-a Mes-Meg T scap & se-z.subm-pont-j.sib & $23,3 \%$ \\
\hline
\end{tabular}




\begin{tabular}{|c|c|c|c|}
\hline Nonnea pulla DC. & v-a Mes-Mac H/T scap bienn & subpont & $16,6 \%$ \\
\hline \multicolumn{4}{|l|}{ BRASSICACEAE } \\
\hline Alliaria petiolata (M.Bieb.) Cavara \& Grande & v-a Meg H scap bienn & ev-med-z.tur & $30 \%$ \\
\hline Alyssum alyssoides (L.) L. & v Mi-Mes T scap & med-subm-pont & $16,6 \%$ \\
\hline Alyssum margrafi O.E. Schulz ex Markgr. & a Mes-Meg H scap & balk (end) & $3,3 \%$ \\
\hline Alyssum turkestanicum Regel \& Schmalh. & v Mi-Mes T scap & pan-pont-j.sib-or-tur & $3,3 \%$ \\
\hline Arabidopsis thaliana (L.) Heynh. & a Mi-Meg H ros bienn/a T ros-scap & ev-med-z.ca & $13,3 \%$ \\
\hline Armoracia rusticana P. Gaertn., B. Mey. \& Scherb. & a meg-Alt G rad scap & adv (pont-kult) & $3,3 \%$ \\
\hline Barbarea vulgaris R. Br. & a Meg H scap & evr (subbor-merid) & $16,6 \%$ \\
\hline Berteroa incana (L.) DC. & a Mes H scap & se-pont-j.sib-tur & $43,3 \%$ \\
\hline Calepina irregularis (Asso) Thell. & a Mes-meg T scap & med-pont-tur & $36,6 \%$ \\
\hline Camelina sativa (L.) Crantz. & a Mes-Alt T scap & evr (temp-merid) & $33,3 \%$ \\
\hline Capsella bursa-pastoris (L.) Medik. & V-aut Mi-Meg T ros/H ros bienn & kosm (subm) & $66,6 \%$ \\
\hline Cardamine hirsuta L. & v-aut Mi-Mes T scap & $\operatorname{kosm}(\mathrm{evr})$ & $33,3 \%$ \\
\hline Cardaria draba (L.) Desv. & v-a Meg H scap & med-subm-pont-tur & $63,3 \%$ \\
\hline Conringia orientalis (L.) Dumort. & v-a Mes-meg T scap & i.med-i.subm-z.pont & $30 \%$ \\
\hline Coronopus squamatus (Forssk.) Asch. & v-a Mi-Mes T rept & $\operatorname{kosm}(\mathrm{med})$ & $20 \%$ \\
\hline Descurainia sophia (L.) Prantl & a Meg T scap/a H scap bienn & evr (temp-merid) & $46,6 \%$ \\
\hline Diplotaxis muralis (L.) DC. & v-a Mes T semiros/H semiros & se-subm & $46,6 \%$ \\
\hline Erophila verna (L.) Chevall. & v N-Mi T ros & med-pont-j.sib-tur & $30 \%$ \\
\hline Erysimum cuspidatum (M.Bieb..) DC. & a Meg T scap/a H scap bienn & i.subm-pan-z.pont-tur & $36,6 \%$ \\
\hline Erysimum diffusum Ehrh. & a Mes-Meg H scap bienn & pont-j.sib-tur & $50 \%$ \\
\hline Lepidium campestre (L.) W.T. Aiton & a Meg T scap/a H scap bienn & ev-subm-pont & $6,6 \%$ \\
\hline Lepidium ruderale L. & a Mes T scap & evr (temp-merid) & $6,6 \%$ \\
\hline Microthlaspi perfoliatum (L.) F. K. Mey. & v Mi-Mes T scap-semiros & med-subm-pont-tur & $23,3 \%$ \\
\hline Myagrum perfoliatum L. & v Mes T scap & i.subm-pont-tur & $26,6 \%$ \\
\hline $\begin{array}{l}\text { Odontarrhena bertolonii subsp. scutarina (Nyár) Španiel, } \\
\text { Al-Shehbaz, D.A. German \& Marhold }\end{array}$ & a Mes-Meg H scap & balk (end) & $30 \%$ \\
\hline Raphanus raphanistrum L. & v-a Meg T scap & evr (temp-merid) & $16,6 \%$ \\
\hline Rorippa austriaca (Crantz) Besser & a Meg H scap & i.med-z.pont & $30 \%$ \\
\hline Rorippa pyrenaica (All.) Rchb. & a Mes H semiros-scap & subm (ev) & $23,3 \%$ \\
\hline Rorippa sylvestris subsp. kerneri (Menyh.) Soó & v-a Mi-Mes H scap bienn & panon & $33,3 \%$ \\
\hline Rorippa silvestris (L.) Besser & a Mi-Mes H scap & med-subm-pan-pont & $43,3 \%$ \\
\hline Sinapis alba L. & v-a Meg T scap & i.med-subm & $46,6 \%$ \\
\hline Sinapis arvensis L. & v-a Mes-Meg T scap & $\operatorname{kosm}($ subm $)$ & $36,6 \%$ \\
\hline
\end{tabular}




\begin{tabular}{|c|c|c|c|}
\hline Sisymbrium loeselii L. & a Meg-Alt T scap & i.subm-pan-pont-tur & $26,6 \%$ \\
\hline Sisymbrium officinale (L.) Scop. & a Meg T scap & med-subm-pont-j.c.sib & $46,6 \%$ \\
\hline Sisymbrium orientale L. & a Meg T scap/ a H scap bienn & med-pont & $40 \%$ \\
\hline Thlaspi arvense L. & a Mes T scap & evr (temp-merid) & $60 \%$ \\
\hline \multicolumn{4}{|l|}{ CANNABACEAE } \\
\hline Humulus lupulus L. & a SH herb & evr-sam (subbor-temp) & $23,3 \%$ \\
\hline \multicolumn{4}{|l|}{ CAPRIFOLIACEAE } \\
\hline Dipsacus laciniatus L. & a Meg-Alt H scap bienn & c.ev-sarm-i.subm-pont-j.sib-or & $16,6 \%$ \\
\hline Knautia arvensis (L.) Coult & a Mes-Meg H scap/a H scap bienn & ev (boreo)-se-med-subm-pont-j.sib & $16,6 \%$ \\
\hline Scabiosa argentea L. & v-a Meg H scap bienn/H scap & i.subm-pan-z.pont & $23,3 \%$ \\
\hline Valerianella carinata Loisel. & v Mi-Mes T scap & atl-se-med-subm & $33,3 \%$ \\
\hline Valerianella locusta (L.) Laterr. & a Mes T scap & kosm (med) & $26,6 \%$ \\
\hline Valerianella turgida (Steven) Betcke & v Mes T scap & i.med-subm & $23,3 \%$ \\
\hline \multicolumn{4}{|l|}{ CARYOPHYLLACEAE } \\
\hline Agrostemma githago L. & a Meg T scap & evr (temp-merid) & $16,6 \%$ \\
\hline Arenaria serpyllifolia L. & v-a Mi-mes T scap & ev-md-pont-tur & $43,3 \%$ \\
\hline Cerastium brachypetalum Pers. & a Mi-Mes T scap & ev-med-subm-z.pont & $20 \%$ \\
\hline $\begin{array}{l}\text { Cerastium fontanum } \\
\text { subsp. vulgare (Hartm.) Greuter \& Burdet }\end{array}$ & v-a Mi-Mes H scap & kosm (evr) & $13,3 \%$ \\
\hline Cerastium glomeratum Thuill. & a Mi-Mes T scap & kosm (med) & $23,3 \%$ \\
\hline Herniaria glabra L. & v-a Mi-Mes T rept/H rept bienn & evr (subbor-submerid) & $26,6 \%$ \\
\hline $\begin{array}{l}\text { Holosteum umbellatum L. } \\
\text { var. umbellatum }\end{array}$ & v Mi-mes T scap & evr (temp-submerid) & $40 \%$ \\
\hline Moenchia mantica (L.) Bartl. & V-a Mes T scap & (ev)-c.i.med-c.i.subm-pan & $20 \%$ \\
\hline Myosoton aquaticum (L.) Moench. & a Mes-Meg H rept & evr (bor-subtemp) & $13,3 \%$ \\
\hline $\begin{array}{l}\text { Petrorhagia saxifraga (L.) Link. } \\
\text { f. cinerascens Th. Wolf. }\end{array}$ & a Mes $H$ caesp & c.i.med-pan-z.pont & $23,3 \%$ \\
\hline Saponaria officinalis L. & a Meg H scap & se-med-pont-j.sib & $16,6 \%$ \\
\hline $\begin{array}{l}\text { Scleranthus annuus L. } \\
\text { subsp. polycarpos (Torn.) Thell. }\end{array}$ & v-aut Mi-Mes T scap/H bienn & evr (temp-submerid) & $10 \%$ \\
\hline Scleranthus perennis subsp. dichotomus (Schur) Nyman & v-aut Mi-Mes H scap & i.subm-pan & $13,3 \%$ \\
\hline Silene armeria L. & a Meg T scap/H scap bienn & evr-sam (temp-submerid) & $20 \%$ \\
\hline $\begin{array}{l}\text { Silene conica L. } \\
\text { subsp. conica Gusul. }\end{array}$ & v-a Mi-Mes T scap & evr (temp-submerid) & $23,3 \%$ \\
\hline Silene flos-cuculi (L.) Clairv. & a Meg H scap & se-ev (bor)-subm-pont-j.sib & $13,3 \%$ \\
\hline
\end{tabular}




\begin{tabular}{|c|c|c|c|}
\hline Silene latifolia Poir. & a Meg H scap bienn/a H scap & evr (temp-submerid) & $30 \%$ \\
\hline Silene vulgaris (Moench) Garcke & a Meg H scap/a G rad & evr (bor-merid) & $50 \%$ \\
\hline Stellaria graminea L. & a Mes-Meg H scap & evr (bor-submerid) & $20 \%$ \\
\hline Stellaria media (L.) Cirillo & v-aut Mi T rept & kosm (med) & $60 \%$ \\
\hline \multicolumn{4}{|l|}{ CHENOPODIACEAE } \\
\hline Atriplex patula L. & aut Meg-Alt T scap & evr-sam (subbor-merid) & $10 \%$ \\
\hline Atriplex rosea L. & a-aut Mes-Meg T scap & med-subm-pan-pont.j.sib-or & $6,6 \%$ \\
\hline Atriplex tatarica L. & a Meg-Alt T scap & evr (temp-merid) & $13,3 \%$ \\
\hline Bassia scoparia (L.) A.J. Scot & a Meg-Alt T scap & $\operatorname{adv}(\mathrm{ca})$ & $3,3 \%$ \\
\hline Blitum bonus-henricus (L.) Rchb. & a Mac H scap & ev-sam (bor-merid) & $6,6 \%$ \\
\hline Chenopodiastrum hybridum (L.) S. Fuentes $\&$ al. & a Mes-Meg T scap & evr (temp-merid) & $13,3 \%$ \\
\hline Chenopodiastrum murale (L.) S. Fuentes \& al. & a Meg-Alt T scap & kosm (med) & $23,3 \%$ \\
\hline $\begin{array}{l}\text { Chenopodium album L. } \\
\text { var. album }\end{array}$ & a Meg-Alt T scap & evr (bor-merid) & $60 \%$ \\
\hline Chenopodium opulifolium W.D.J. Koch \& Ziz & a Meg T scap & kosm (med) & $43,3 \%$ \\
\hline Chenopodium strictum Roth. & a Meg T scap & ev-z.az & $33,3 \%$ \\
\hline $\begin{array}{l}\text { Chenopodium suecicum Murr } \\
\text { var. viride (L.) Wahlenb }\end{array}$ & a Mes-Alt T scap & evr (temp-submerid) & $26,6 \%$ \\
\hline Dysphania botrys (L.) Mosyakin \& Clemants & a Mes-Meg T scap & evr (temp-merid) & $43,3 \%$ \\
\hline Lipandra polysperma ( L.) S. Fuentes \& al. & a Meg T scap & evr & $10 \%$ \\
\hline Salsola tragus L. subsp. tragus & a Mes-Alt T scap & pan-pont-j.sib-tur-ca & $3,3 \%$ \\
\hline \multicolumn{4}{|l|}{ CISTACEAE } \\
\hline Fumana procumbens (Dunal.) Gren.\& Godr. & a Mes Ch suff rept & z.i.c.med-subm-se-z.pont-or & $13,3 \%$ \\
\hline \multicolumn{4}{|l|}{ CONVOLVULACEAE } \\
\hline Calystegia sepium (L.) R.Br. & a SH herb & kosm (evr-sam) & $16,6 \%$ \\
\hline Convolvulus arvensis L. & a SG herb rhiz & kosm (med) & $93,3 \%$ \\
\hline Cuscuta epithymum (L.) L. & ST par & atl-se-med-subm-pan-pont-j.sib & $23,3 \%$ \\
\hline Cuscuta europaea L. & ST par & ev-az & $30 \%$ \\
\hline \multicolumn{4}{|l|}{ CRASSULACEAE } \\
\hline Sedum acre L. & N-Mi Ch herb caesp succ & atl-se-med & $6,6 \%$ \\
\hline \multicolumn{4}{|l|}{ CUCURBITACEAE } \\
\hline Bryonia alba L. & a SG tub herb & sarm-i.subm-pont-j.sib & $13,3 \%$ \\
\hline Echinocystis lobata (Michx.) Torr.\& A. Gray & a St herb & $\operatorname{adv}(\mathrm{sam})$ & $10 \%$ \\
\hline \multicolumn{4}{|l|}{ CYPERACEAE } \\
\hline Carex hirta L. & a Mes-Meg G rhiz caesp & ev-med-pont & $23,3 \%$ \\
\hline
\end{tabular}

APPLIED ECOLOGY AND ENVIRONMENTAL RESEARCH 15(4):863-890.

http://www.aloki.hu • ISSN 15891623 (Print) • ISSN 17850037 (Online)

DOI: http://dx.doi.org/10.15666/aeer/1504 863890

(c) 2017, ALÖKI Kft., Budapest, Hungary 


\begin{tabular}{|c|c|c|c|}
\hline Carex panicea L. & v-a Meg H caesp/rhiz & cirkumpolarni & $20 \%$ \\
\hline $\begin{array}{l}\text { Carex vulpina L. } \\
\text { f. nemorosa (Rebent.) Koch. }\end{array}$ & a Meg H caesp & evr (temp-submerid) & $30 \%$ \\
\hline Cyperus fuscus L. & a Mes emer Hyd T scap & evr (subbor-merid) & $16,6 \%$ \\
\hline Eleocharis palustris (L.) R.Br. & a Mes-Meg emer Hyd G rhiz & kosm (evr) & $16,6 \%$ \\
\hline Shoenoplectus lacuster (L.) Palla & a-aut Alt emer Hyd G rhiz & kosm & $13,3 \%$ \\
\hline \multicolumn{4}{|l|}{ EQUISETACEAE } \\
\hline Equisetum arvense L. & a Mes-Meg G rhiz scap & evr-sam (bor-temp) & $20 \%$ \\
\hline Equisetum palustre L. & a Meg G rhiz & evr-sam (bor-temp) & $13,3 \%$ \\
\hline \multicolumn{4}{|l|}{ EUPHORBIACEAE } \\
\hline Euphorbia cyparissias L. & a Mes-Meg H scap & atl-se-c.subm-pan-sarm & $50 \%$ \\
\hline Euphorbia taurensis All. & a Mes T scap & subm & $33,3 \%$ \\
\hline Euphorbia helioscopia L. & a Mi-Meg T scap & kosm (evr) & $9-30 \%$ \\
\hline $\begin{array}{l}\text { Euphorbia seguieriana Neck. } \\
\text { subsp. niciciana (Borbas ex Novak) Reich. }\end{array}$ & a Mes-Meg G rad caesp & evr (temp-submerid) & $40 \%$ \\
\hline Euphorbia stricta L. & v-a Mes-Meg T scap/H bienn & ev-z.az. & $26,6 \%$ \\
\hline \multicolumn{4}{|l|}{ FABACEAE } \\
\hline Amorpha fruticosa L. & fo dec Mi P caesp & adv (s.amer) & $6,6 \%$ \\
\hline Anthyllis vulneraria L. & a Mes-Meg H scap & ev-med-subm-z.pont & $3,3 \%$ \\
\hline $\begin{array}{l}\text { Astragalus cicer L. } \\
\text { f. microphyllus (L.) Acherson et Graebn. }\end{array}$ & a Mes-Meg H scap & j.atl-subm-pont-sarm & $6,6 \%$ \\
\hline Astragalus glycyphyllos L. & a Mes-Meg H scap-rept & ev.i-subm-pont-j.sib-tur & $13,3 \%$ \\
\hline $\begin{array}{l}\text { Astragalus hamosus L. } \\
\text { f. multiflorus }\end{array}$ & v-a Mes-Meg T scap & med-subm-tur & $3,3 \%$ \\
\hline $\begin{array}{l}\text { Dorycnium pentaphyllum subsp. herbaceum (Vill.) Bonnier } \\
\text { \& Layens }\end{array}$ & Mes Ch suff caesp & med (ap-balk) c.subm-pan-pont & $23,3 \%$ \\
\hline Galega officinalis L. & a Meg H scap & c.i.subm-pan-pont-or & $13,3 \%$ \\
\hline Genista tinctoria L. & Meg fo dec Ch suff caesp & se-sarm-subm-pont-j.c.sib & $26,6 \%$ \\
\hline Lathyrus aphaca L. & a Mes T scap/ST herb & med-subm-pont-j.tur & $33,3 \%$ \\
\hline Lathyrus sphaericus Retz. & a Mes-Meg T scap & med-subm & $23,3 \%$ \\
\hline Lathyrus tuberosus L. & a Meg G tub rept & se-subm-pont-j.sib-or-i.afr & $36,6 \%$ \\
\hline Lotus corniculatus L. & a Mes H scap & ev-med-pont-j.sib-or-i.afr & $66,6 \%$ \\
\hline Medicago arabica (L.) Huds. & a Meg T scap & med-subm-or-tur & $56,6 \%$ \\
\hline Medicago falcata L. & a Mes-Meg H scap & ev-med-subm-pont-j.sib & $40 \%$ \\
\hline Medicago lupulina L. & a Mes T scap/a H scap & evr (temp-merid)-i.afr & $53,3 \%$ \\
\hline
\end{tabular}


Medicago minima (L.) L.

Medicago orbicularis (L.) Bartal.

Medicago rigidula (L.) All.

Medicago sativa L.

Melilotus albus Medik.

Melilotus officinalis (L.) Lam.

Onobrychis viciifolia Scop.

Ononis spinosa L.

Ononis spinosa subsp. hircina (Jacq.) Gams

Robinia pseudoacacia L.

Securigera varia (L.) Lassen

Trifolium arvense L.

Trifolium campestre Schreb.

Trifolium dubium Sibth.

Trifolium fragiferum $\mathrm{L}$.

Trifolium incarnatum L.

Trifolium patens Schreb.

Trifolium pratense L.

Trifolium repens L.

Trifolium resupinatum L.

Vicia cracca L.

var. linearis Petern

Vicia grandiflora Scop.

Vicia hirsuta (L.) Gray

f. fissa (Frol) Beck.

Vicia lutea L.

Vicia pannonica Crantz

Vicia pannonica subsp. striata (M. Bieb.) Nyman

Vicia sativa L.

Vicia sativa subsp. nigra (L.) Ehrh.

Vicia tenuifolia Roth.

Vicia tetrasperma (L.) Schreb.

Vicia villosa Roth.

FUMARIACEAE

Fumaria officinalis L. a Mi-Mes T scap

a Mes T scap

a Mi-Mes T scap

a Mes-Meg H scap

a Meg T scap/a H scap bienn

a Meg-Alt H scap bienn

a Meg H scap

fo dec Mes-Meg Ch suff caesp

fo dec Mes Ch suff caesp

fo dec Mes P scap

a Meg H scap

a Mes T scap/a H scap bienn

a Mes T scap

v-a Mi-Mes T scap

a Mes H rept

a Mes-Meg T scap

a Mes H scap

a Mi H rept

a Mes T scap

a Meg-Alt H scap/SH herb

a Meg T scap/ST herb

a Mes-Meg T scap/ST herb

v-a Mes-Meg T scap

a Mes-Meg T scap/ST herb

a Mes-Meg T scap

a Mes-Meg T scap/ST herb

v-a Mes-Meg T scap/H scap bienn

a Mac-Alt H scap/SH herb

Mes ST herb

a Meg-Alt T scap/ST herb

a Mi-Mes T scap a Mes-Meg T scap

\begin{tabular}{|l|l|}
\hline evr (submerid-merid)-i.afr. & $30 \%$ \\
\hline med-subm-or-tur & $43,3 \%$ \\
\hline med-subm-or & $46,6 \%$ \\
\hline med-subm-or & $33,3 \%$ \\
\hline ev-subm-pont-j.sib-or-tur & $43,3 \%$ \\
\hline adv (med;kult) & $60 \%$ \\
\hline atl-c.ev & $16,6 \%$ \\
\hline i.subm-pan-sarm-pont-j.sib & $23,3 \%$ \\
\hline adv (sam) & $20 \%$ \\
\hline se-med-subm-pont-or & $13,3 \%$ \\
\hline ev-med-pont-j.sib & $30 \%$ \\
\hline ev-med-subm-z.pont-or-tur & $40 \%$ \\
\hline ev-z.az & $50 \%$ \\
\hline ev-med-subm-pont-j.sib-or-tur & $70 \%$ \\
\hline subatl-med-subm & $60 \%$ \\
\hline (ev) med-subm & $40 \%$ \\
\hline ev-med-subm-pont-j.sib & $63,3 \%$ \\
\hline kosm (evr) & $33,3 \%$ \\
\hline med-subm-or-tur & $50 \%$ \\
\hline evr (bor-merid) & $36,6 \%$ \\
\hline i.subm-pan-z.pont & $53,3 \%$ \\
\hline ev-med-pont-j.sib & \\
\hline z.c.med-subm-or-tur & $13,3 \%$ \\
\hline ev-med-subm-z.pont & $43,3 \%$ \\
\hline i.med-i.subm-or & \\
\hline kosm (med;kult) & $40 \%$ \\
\hline ev-z.az & $43,3 \%$ \\
\hline ev-med-z.az & $43,3 \%$ \\
\hline kosm (med) & $50 \%$ \\
\hline c.i.subm-sarm-pont & $36,6 \%$ \\
\hline ev-med-subm-pont-or & $30 \%$ \\
\hline & $26,6 \%$ \\
\hline & $63,3 \%$ \\
\hline & $53,3 \%$ \\
\hline & \\
\hline & \\
\hline & \\
\hline
\end{tabular}

APPLIED ECOLOGY AND ENVIRONMENTAL RESEARCH 15(4):863-890.

http://www.aloki.hu • ISSN 15891623 (Print) • ISSN 17850037 (Online)

DOI: http://dx.doi.org/10.15666/aeer/1504 863890

(c) 2017, ALÖKI Kft., Budapest, Hungary 


\begin{tabular}{|c|c|c|c|}
\hline \multicolumn{4}{|l|}{ GERANIACEAE } \\
\hline Erodium cicutarium (L.) L' Hér. & v-a Mi-Mes T semiros-scap & evr (submerid-merid) & $50 \%$ \\
\hline Geranium columbinum L. & a Mi-Meg T scap & atl.c.ev-med-subm-pan-or & $40 \%$ \\
\hline Geranium dissectum L. & a Mi-Meg T scap & atl-c.ev-med-subm-pan-or & $46,6 \%$ \\
\hline Geranium molle L. & a Mi-mes T scap/a H scap bienn & se-sarm-med-subm & $50 \%$ \\
\hline Geranium pyrenaicum Burm. & a Mes-Meg H scap & atl-c.ev-med-subm & $36,6 \%$ \\
\hline \multicolumn{4}{|l|}{ HYPERICACEAE } \\
\hline Hypericum perforatum L. & a Mes-Meg H scap & se-med-pont-j.sr.sib-or-tur & $23,3 \%$ \\
\hline \multicolumn{4}{|l|}{ JUNCACEAE } \\
\hline $\begin{array}{l}\text { Juncus articulatus L. } \\
\text { subsp.articulatus }\end{array}$ & a Mes-Meg G rhiz caesp & evr-sam (bor-merid) & $20 \%$ \\
\hline \multicolumn{4}{|l|}{ LAMIACEAE } \\
\hline Ajuga genevensis L. & a Mi-Mes H semiros & se-subm-pont & $50 \%$ \\
\hline Ajuga reptans L. & a Mes H rept & se-med-subm & $33,3 \%$ \\
\hline Ballota nigra L. & a Meg H scap & se-med-subm-pont-or-tur & $50 \%$ \\
\hline Galeopsis ladanum L. & a Mes-Meg T scap & evr (temp-submerid)-sam (sin) & $43,3 \%$ \\
\hline Glechoma hederacea L. & a Mes-Meg H rept/Ch herb rept & evr (subor-submerid) & $50 \%$ \\
\hline Lamium amplexicaule L. & v Mi-Mes T scap & evr (temp-submerid)-i.afr & $70 \%$ \\
\hline Lamium maculatum (L.) L. & v-a Mes-Meg H scap & se-subm-z.pont & $66,6 \%$ \\
\hline Lamium purpureum L. & v Mi-Mes T scap & se-med-subm-pont & $63,3 \%$ \\
\hline Leonurus cardiaca L. & a Meg-Alt H scap & sarm-subm-pont-j.c.sir-or-tur & $23,3 \%$ \\
\hline Lycopus europaeus L. & a Mes-Meg H scap/emer Hyd G rhiz & evr (subbor-merid) & $26,6 \%$ \\
\hline Marrubium peregrinum L. & a Meg-Alt H scap & balk-pan-z.pont & $23,3 \%$ \\
\hline Mentha aquatica L. & a Mes-Meg H scap & evr (subbor-submerid)-afr(boreosubtrop) & $6,6 \%$ \\
\hline Mentha longifolia (L.) Huds. & a Mes-Meg H scap & evr (temp-merid)-afr (boreosubtrop) & $6,6 \%$ \\
\hline Prunella vulgaris L. & a Mi-Mes H scap-semiros & evr-sam (subbor-submerid) & $20 \%$ \\
\hline Salvia amplexicaulis Lam. & a Meg-Alt H scap & i.med-i.subm & $36,6 \%$ \\
\hline Salvia nemorosa L. & a Mes-Meg H scap & sarm-i.subm-pont-j.sib-or-tur & $30 \%$ \\
\hline Salvia pratensis L. & a Mes-Meg H scap & subm-pont-j.sib & $43,3 \%$ \\
\hline Salvia verticillata $\mathrm{L}$. & a Mes-Meg H scap & se-subm-pont-j.sib & $23,3 \%$ \\
\hline Scutellaria galericulata L. & a Mi-Meg G rhiz scap & evr-sam (bor-temp) & $16,6 \%$ \\
\hline Stachys palustris L. & a Mes-Meg H scap & evr-sam (bor-submerid) & $13,3 \%$ \\
\hline Teucrium chamaedrys L. & Mes Ch suff caesp & med-subm-z.pont-or & $20 \%$ \\
\hline Thymus longicaulis C. Presl. & v-a Mi-Mes Ch herb rept & balk (end.) & $16,6 \%$ \\
\hline
\end{tabular}




\begin{tabular}{|c|c|c|c|}
\hline \multicolumn{4}{|l|}{ LILIACEAE } \\
\hline Ornithogalum umbellatum L. & v Mi G bulb scap & se-med-subm-pan-z.pont & $13,3 \%$ \\
\hline \multicolumn{4}{|l|}{ LINACEAE } \\
\hline Linum austriacum L. & a Mi-Meg H scap & subm-pan-pont-(herc) & $23,3 \%$ \\
\hline \multicolumn{4}{|l|}{ LYTHRACEAE } \\
\hline $\begin{array}{l}\text { Lythrum salicaria L. } \\
\text { var. tomentosum (Mill.) D }\end{array}$ & a Meg-Alt H scap & kosm (evr) & $16,6 \%$ \\
\hline MALVACEAE & & & $13,3 \%$ \\
\hline Althaea hirsuta L. & a Mes-Meg T scap & med-subm-pan-pont-or & $10 \%$ \\
\hline Alcea rosea L. & a Meg-Alt H scap & $\operatorname{adv}$ (kult) & $10 \%$ \\
\hline Malva neglecta Wallr. & a Mes-Meg T scap & med-pont-or-tur & $20 \%$ \\
\hline Malva pusilla Sm. & a-aut Mes-Meg T scap & evr (temp-submerid) & $13,3 \%$ \\
\hline Malva sylvestris L. & a Meg-Alt H scap bienn/a H scap & kosm (evr) & $13,3 \%$ \\
\hline \multicolumn{4}{|l|}{ MORACEAE } \\
\hline Maclura pomifera (Raf.) C.K. Schneid. & fo dec Mes P scap & adv (sam, kult) & $3,3 \%$ \\
\hline \multicolumn{4}{|l|}{ ONAGRACEAE } \\
\hline Epilobium angustifolium L. & a Meg-Alt H scap & evr-sam (bor-submerid) & $10 \%$ \\
\hline Epilobium hirsutum L. & a Mes-Meg H scap & evr (subbor-merid)-afr (boreo-austrosubtrop) & $10 \%$ \\
\hline \multicolumn{4}{|l|}{ OLEACEAE } \\
\hline Fraxinus ornus L. & fo dec Mes P scap & c.med-i.subm & $6,6 \%$ \\
\hline Ligustrum vulgare L. & fo dec NP caesp & atl-se-med-subm-or & $13,3 \%$ \\
\hline \multicolumn{4}{|l|}{ OROBANCHACEAE } \\
\hline Melampyrum arvense L. & a Mes-Meg T scap & evr (temp-submerid) & $16,6 \%$ \\
\hline Rhinanthus major L. & v-a Mes-Meg T scap & se & $20 \%$ \\
\hline \multicolumn{4}{|l|}{ PAPAVERACEAE } \\
\hline Chelidonium majus L. & v-a Mes-Meg H semiros & evr (temp-submerid) & $36,6 \%$ \\
\hline Papaver dubium L. & a Meg T scap & atl-se-med-subm-z.pont-or-afr (boreosubtrop) & $56,6 \%$ \\
\hline Papaver rhoeas L. & a Meg t scap & ev-med-subm-pont-j.sib-or & $46,6 \%$ \\
\hline \multicolumn{4}{|l|}{ PHYTOLACCACEAE } \\
\hline Phytolacca americana L. & a-aut Alt G rhiz scap & adv (sam) & $3,3 \%$ \\
\hline \multicolumn{4}{|l|}{ PLANTAGINACEAE } \\
\hline Plantago lanceolata L. & a Mi-Meg H ros & evr (subbor-temp) & $66,6 \%$ \\
\hline Plantago major L. & a Mes-Meg H ros & kosm (evr-sam) & $50 \%$ \\
\hline Plantago media L. & a Mes-Meg H ros & evr (temp-submerid) & $46,6 \%$ \\
\hline
\end{tabular}




\begin{tabular}{|c|c|c|c|}
\hline \multicolumn{4}{|l|}{ PLATANACEAE } \\
\hline Platanus orientalis L. & fo dec Mes P scap & adv (?, kult) & $6,6 \%$ \\
\hline \multicolumn{4}{|l|}{ POACEAE } \\
\hline Achnatherum calamagrostis (L.) P. Beauv. & a Meg-Alt H scap & JEP (južnoevropsko planinska) & $23,3 \%$ \\
\hline Aegilops cylindrica Host & a Mi-Mes T scap & i.med-subm-pont-or-tur & $43,3 \%$ \\
\hline Aegilops neglecta Bertol. & v-a Mes T caesp & med-subm-tur & $40 \%$ \\
\hline Alopecurus myosuroides Huds. & a Mes-Meg T caesp & atl-med-subm-or-tur & $30 \%$ \\
\hline Alopecurus pratensis L. & a Meg-Alt H caesp & evr (subbor-submerid) & $23,3 \%$ \\
\hline Anisantha sterilis (L.) Nevski & a Mes-Meg T caesp & se-sarm-med-subm-pont-or & $53,3 \%$ \\
\hline Anisantha tectorum (L.) Nevski & a Mes-Meg T scap & ev-med-subm-pont-j.sib-or-tur & $40 \%$ \\
\hline Anthoxanthum odoratum L. & a Mes-Meg H caesp & ev-med-subm-z.pont-sr.sib & $23,3 \%$ \\
\hline Apera spica-venti (L.) P. Beauv. & a Mes-Meg T scap & ev-subm-pont-j.sr.sib & $26,6 \%$ \\
\hline Arrhenatherum elatius (L.) J. Presl \& C. Presl & a Meg-Alt H caesp & atl-se-subm-sarm & $30 \%$ \\
\hline Botriochloa ishaemum (L.) Keng. & a Mes H caesp & evr (temp-merid) & $13,3 \%$ \\
\hline Briza media L. & a Mes-Meg H caesp & evr (subbor-submerid) & $20 \%$ \\
\hline Bromus arvensis L. & a Mes-Meg T scap & subm-sarm-sr.sib & $50 \%$ \\
\hline Bromus commutatus Schrad. & a Meg T scap & subatl-se-c.subm & $46,6 \%$ \\
\hline Bromus hordeaceus L. & & & $40 \%$ \\
\hline Bromus inermis (Leyss.) Holub & a Meg-Alt H caesp & evr (subbor-temp)-sam(temp-submerid) & $30 \%$ \\
\hline Bromus racemosus L. & v-a Mes-Meg T scap & ev-z.az & $43,3 \%$ \\
\hline Bromus squarrosus L. & a Mes-Meg T scap & med-subm-pan-pont-j.sib-or-tur & $53,3 \%$ \\
\hline Cynodon dactylon (L.) Pers. & a Mes G rhiz rept-caesp & kosm (med-or-tur) & $53,3 \%$ \\
\hline Dactylis glomerata L. & a Meg H caesp & ev-med-subm-pont-j.sib-or-tur-ca & $30 \%$ \\
\hline Dasypyrum villosum (L.) P. Candargy & a Mes-Meg T scap & med-subm & $33,3 \%$ \\
\hline Digitaria sanguinalis (L.) Scop. & a Mes T caesp-rept & evr-sam (subbor-merid) & $23,3 \%$ \\
\hline Echinochloa crus-galli (L.) P. Beauv. & a Meg-Alt T caesp & kosm (subtrop-trop) & $26,6 \%$ \\
\hline Eragrostis pilosa (L.) P. Beauv. & a Mes-Meg T caesp & $\operatorname{kosm}(\mathrm{med})$ & $20 \%$ \\
\hline Elytrigia repens (L.) Nevski & a Mes-Meg G rhiz caesp & kosm (evr) & $16,6 \%$ \\
\hline $\begin{array}{l}\text { Festuca rubra L. } \\
\text { subsp. rubra }\end{array}$ & a Mes-Meg H caesp & evr-sam (bor-merid) & $26,6 \%$ \\
\hline Festuca valesiaca Gaudin & a Meg H caesp & ev-subm-pont-j.sib-ca & $20 \%$ \\
\hline Glyceria fluitans (L.) R.Br. & v-aut Meg-Alt Hyd G rhiz & evr (bor-submerid)-sam & $13,3 \%$ \\
\hline Holcus lanatus L. & a Meg H caesp & atl-med-se-sarm-z.pont & $30 \%$ \\
\hline Hordeum murinum L. & a Mes T caesp & med-subm-sarm-z.pont & $60 \%$ \\
\hline Hordeum murinum L. & a Mes T caesp & med-subm-sarm-z.pont & $53,3 \%$ \\
\hline
\end{tabular}




\begin{tabular}{|c|c|c|c|}
\hline \multicolumn{4}{|l|}{ subsp. leporinum (Link) Arcang. } \\
\hline Koeleria macrantha (Lebed.) Schult. & a Mes H scap & evr-sam (temp-submerid) & $13,3 \%$ \\
\hline Lolium perenne L. & a Mes H caesp & ev-med-subm & $56,6 \%$ \\
\hline Lolium remotum Schrank & a Mes-Alt T scap & evr (temp) & $53,3 \%$ \\
\hline Melica ciliata L. & a Mes-Meg H caesp & se-med-subm (ev) & $16,6 \%$ \\
\hline Ochlopoa annua (L.) H. Scholz. & v-aut N-Mes T caesp & kosm (evr-sam) & $43,3 \%$ \\
\hline Pennisetum glaucum (L.) R.Br. & a-aut Mes-Meg T caesp & kosm (evr-sam) & $20 \%$ \\
\hline Phragmites australis (Cav.)Trin ex Steud. & Alt emer Hyd G rhiz & kosm (evr-sam) & $6,6 \%$ \\
\hline $\begin{array}{l}\text { Poa bulbosa L. } \\
\text { subsp.. vivipara (Koeler) Arcang. }\end{array}$ & a Mes-Meg H scap & ev-med-subm-pont-j.sib-or-tur-ca & $23,3 \%$ \\
\hline Poa compressa L. & a Mes H caesp & kosm (evr) & $26,6 \%$ \\
\hline Poa nemoralis L. & a Mes-Alt H scap & evr-sam (bor-submerid) & $53,3 \%$ \\
\hline Poa angustifolia L. & a Mes-Meg H caesp & kosm (evr-sam) & $36,6 \%$ \\
\hline Poa trivialis L. & a Mes-Meg H caesp & kosm (evr) & $50 \%$ \\
\hline Schenodorus arundinaceus (Schreb.) Dumort & a Meg-Alt H caesp & subm-pont-j.sib-tur & $26,6 \%$ \\
\hline Schenodorus pratensis (Huds) P. Beauv. & a Meg H caesp & ev-subm-pont-j.sib-ca & $30 \%$ \\
\hline Sclerochloa dura (L.) P. Beauv. & v N-Mi T caesp & med-subm-pont-or-tur & $40 \%$ \\
\hline Setaria viridis (L.) P. Beauv. & a-aut Mes-Meg T caesp & kosm (evr-sam) & $36,6 \%$ \\
\hline Sorghum halepense (L.) Pers. & a-aut Meg-Alt G rhiz caesp & adv (paleotrop) & $33,3 \%$ \\
\hline Vulpia myurus (L.) C.C.Gmel. & a Mes-Meg T caesp & se-med-subm-z.pont-or-tur-ca & $13,3 \%$ \\
\hline \multicolumn{4}{|l|}{ POLYGONACEAE } \\
\hline Fallopia convolvulus (L.) Á. Löve & a Mes-Meg T scap/SH herb & kosm (evr) & $23,3 \%$ \\
\hline Fallopia dumetorum (L.) Holub & a Meg T scap/SH herb & evr (temp-submerid) & $13,3 \%$ \\
\hline Persicaria lapathifolia (L.) Delarbre & a-aut Meg T scap & evr (bor-trop)-sam (subbor-boreotrop) & $6,6 \%$ \\
\hline Persicaria maculosa Gray & a-aut Meg T scap & evr (bor-merid) & $6,6 \%$ \\
\hline Polygonum aviculare L. & a-aut Mi-Meg T rept & kosm (trop) & $53,3 \%$ \\
\hline Rumex acetosella L. & a Mes-Meg H scap & evr-sam (bor-merid) & $40 \%$ \\
\hline Rumex crispus L. & a Meg-Alt H scap & kosm (evr) & $30 \%$ \\
\hline Rumex obtusifolius L. & a Meg H scap & se-subm-pont & $20 \%$ \\
\hline Rumex pulcher L. & v-a Mes-meg H scap/v-a T scap & med-subm-or & $36,6 \%$ \\
\hline \multicolumn{4}{|l|}{ PORTULACACEAE } \\
\hline Portulaca oleracea L. & a Mes T scap & $\operatorname{adv}(\mathrm{az})$ & $10 \%$ \\
\hline \multicolumn{4}{|l|}{ PRIMULACEAE } \\
\hline Anagallis arvensis L. & v-aut Mi T rept & kosm (med) & $16,6 \%$ \\
\hline Anagallis foemina Mill. & V-aut Mi T rept & se-med-subm-z.pont & $6,6 \%$ \\
\hline
\end{tabular}




\begin{tabular}{|c|c|c|c|}
\hline \multicolumn{4}{|l|}{ RANUNCULACEAE } \\
\hline Adonis flammea Jacq. & a Mes-Meg T scap & subm-se-pan-z.pont-or & $23,3 \%$ \\
\hline Clematis vitalba L. & a dec S lig & se-med-subm & $33,3 \%$ \\
\hline Consolida orientalis (J.Gay.)Schrödinger & a Meg T scap & med-or-tur-z.pont & $30 \%$ \\
\hline Consolida regalis Gray & a Mes-Meg T scap & se-subm-pont-j.sib & $20 \%$ \\
\hline Ficaria verna Huds. & v Mi-Mes G scap & evr (bor-submerid) & $16,6 \%$ \\
\hline Nigella damascena L. & a Mes T scap & med-subm-or & $6,6 \%$ \\
\hline Ranunculus arvensis L. & a Mes-Meg T scap-semiros & se-med-or-tur-ca & $36,6 \%$ \\
\hline Ranunculus millefoliatus Vahl & v-a Mes H scap/G tub & c.i.med-subm & $20 \%$ \\
\hline Ranunculus repens L. & a Mes-Meg H rept & evr (bor-submerid) & $40 \%$ \\
\hline Ranunculus sardous Crantz & a Mes-Meg T scap-semiros & se-med-subm & $13,3 \%$ \\
\hline Ranunculus sceleratus L. & a Mes-Meg emer Hyd T semiros & kosm (evr) & $16,6 \%$ \\
\hline Ranunculus serbicus Vis. & a Meg H scap/G rhiz & sr.balk.j.apen (subend) & $13,3 \%$ \\
\hline \multicolumn{4}{|l|}{ RESEDACEAE } \\
\hline Reseda lutea L. & a Mes-Meg H scap/a T scap & se-med-subm-pont-or & $23,3 \%$ \\
\hline Reseda luteola L. & a Mes-Alt H scap bienn/T scap & atl-se-med-subm-or & $26,6 \%$ \\
\hline Reseda phyteuma L. & v-a Mes-Mac T scap/H scap & med-subm & $6,6 \%$ \\
\hline \multicolumn{4}{|l|}{ ROSACEAE } \\
\hline Agrimonia eupatoria L. & a Meg H scap & ev-med-subm-or-pont-j.sib-tur & $30 \%$ \\
\hline Crataegus monogyna Jacq. & fo dec NP caesp & se-med-subm-pont & $3,3 \%$ \\
\hline Filipendula vulgaris Moench & a Meg H scap & evr (subbor-submerid) & $10 \%$ \\
\hline Fragaria vesca L. & a Mes H rept & evr (subbor-submerid)-sam (temp) & $16,6 \%$ \\
\hline Geum urbanum L. & a Meg H scap & se-pont-j.sr.sib-tur & $23,3 \%$ \\
\hline Potentilla argentea L. & a Mes-Meg H scap & evr (temp-submerid) & $26,6 \%$ \\
\hline Potentilla hirta L. & a Mes-Meg H scap & (ev) med-subm-z.pont & $26,6 \%$ \\
\hline Potentilla neglecta Baumg. & a Mes-Meg H scap & evr (temp-submerid) & $16,6 \%$ \\
\hline Potentilla reptans L. & a Mi-Mes H rept & kosm (evr) & $43,3 \%$ \\
\hline Rosa canina L. & fo dec NP caesp & ev-med-subm-pont-or-tur & $33,3 \%$ \\
\hline Rubus caesius L. & fo dec NP rept & evr (temp-submerid) & $30 \%$ \\
\hline Rubus ulmifolius Schott & fo dec NP rept & atl.z.c.med-subm & $20 \%$ \\
\hline Sanguisorba minor Scop. & a Mes-Meg H scap & evr (temp-submerid) & $50 \%$ \\
\hline \multicolumn{4}{|l|}{ RUBIACEAE } \\
\hline Cruciata laevipes Opiz & v-a Mes-Meg H scap & evr (temp-submerid) & $36,6 \%$ \\
\hline Galium aparine L. & Mes-Meg ST herb & kosm (evr) & $50 \%$ \\
\hline Galium lucidum All. & a Mes-Meg H scap & med-subm-se & $30 \%$ \\
\hline
\end{tabular}

APPLIED ECOLOGY AND ENVIRONMENTAL RESEARCH 15(4):863-890.

http://www.aloki.hu • ISSN 15891623 (Print) • ISSN 17850037 (Online)

DOI: http://dx.doi.org/10.15666/aeer/1504_863890

(c) 2017, ALÖKI Kft., Budapest, Hungary 


\begin{tabular}{|c|c|c|c|}
\hline Galium mollugo L. & a Meg-Alt H scap & se-med-subm & $40 \%$ \\
\hline Galium verum L. & a Mes-Meg H scap & kosm (med) & $23,3 \%$ \\
\hline Sherardia arvensis L. & v-a Mi-Mes T scap & kosm (med) & $16,6 \%$ \\
\hline \multicolumn{4}{|l|}{ SALICACEAE } \\
\hline Populus alba L. & fo dec Mes P scap & se-med-subm-pont-j.sib-tur & $16,6 \%$ \\
\hline Populus nigra L. & fo dec Mes P scap & se-med-subm-pont-j.sib & $16,6 \%$ \\
\hline Salix alba L. & fo dec Mes P scap & ev-med-subm-or-pont-j.c.sib-ca & $16,6 \%$ \\
\hline Salix triandra L. & fo dec Mes P caesp-scap & evr (bor-submerid) & $16,6 \%$ \\
\hline \multicolumn{4}{|l|}{ SANTALACEAE } \\
\hline Comandra umbellata (L.) Nutt. & v-a Mes-Meg Ch frut/Ch suffr & subdac & $3,3 \%$ \\
\hline Thesium arvense Horv. & a Mes H scap/H bienn & evr (temp-submerid) & $13,3 \%$ \\
\hline \multicolumn{4}{|l|}{ SAPINDACEAE } \\
\hline Acer negundo L. & fo dec Mes P scap & $\operatorname{adv}(\mathrm{sam})$ & $10 \%$ \\
\hline Acer pseudoplatanus L. & fo dec Mes P scap & $\operatorname{adv}(\mathrm{sam})$ & $10 \%$ \\
\hline Acer saccharinum L. & fo dec Mes P scap & se & $10 \%$ \\
\hline \multicolumn{4}{|l|}{ SCROPHULARIACEAE } \\
\hline Antirrhinum majus L. & Mes-Meg Ch suff caesp & adv (med-subm, kult) & $6,6 \%$ \\
\hline $\begin{array}{l}\text { Linaria genistifolia subsp. sofiana (Velen.) Chater \& } \\
\text { D.A.Webb }\end{array}$ & a Mes-Meg H scap & balk (end) & $43,3 \%$ \\
\hline Linaria vulgaris Mill. & a-aut Mes-Meg H scap & evr (subbor-submerid) & $23,3 \%$ \\
\hline Verbascum phlomoides L. & a Meg-Alt H ros bienn & se-med (ev)-subm (ev)-pan-z.pont & $56,6 \%$ \\
\hline Veronica anagallis-aquatica L. & a Mes-Meg H scap & kosm (evr) & $16,6 \%$ \\
\hline Veronica arvensis L. & v-a N-Mes T scap & kosm (med) & $20 \%$ \\
\hline Veronica beccabunga L. & v-a Mes-Meg H rept & evr (bor-submerid) & $23,3 \%$ \\
\hline Veronica chamaedrys L. & v-a Mi-Mes H scap & se-subm-pont-j.sib & $43,3 \%$ \\
\hline Veronica hederifolia L. & v Mi-Mes T scap & se-med-subm-pont & $30 \%$ \\
\hline Veronica persica Poir. & V-aut N-Mes T scap & kosm (med-subm) & $26,6 \%$ \\
\hline Veronica praecox All. & v-a Mi-Mes T scap bienn & pont-subm & $36,6 \%$ \\
\hline Veronica serpyllifolia L. & v-aut Mi-Mes H rept & kosm (evr) & $46,6 \%$ \\
\hline \multicolumn{4}{|l|}{ SOLANACEAE } \\
\hline Datura stramonium L. & a-aut Meg-Alt T scap & kosm (evr-sam) & $23,3 \%$ \\
\hline Hyoscyamus niger L. & a-aut Mes-Meg T scap/H scap bienn & evr (temp-merid) & $20 \%$ \\
\hline Lycium barbarum L. & fo dec NP caesp & $\operatorname{adv}(\mathrm{med})$ & $3,3 \%$ \\
\hline Physalis alkekengi L. & a-aut Mes-Meg G rhiz rept & se-med-subm-z.pont & $3,3 \%$ \\
\hline Solanum dulcamara L. & a Meg-Alt S lig & evr (temp-submerid) & $23,3 \%$ \\
\hline
\end{tabular}




\begin{tabular}{|l|l|l|}
\hline Solanum nigrum L. & v-aut Mes-Meg T scap & kosm (evr-sam) \\
\hline TYPHACEAE & & \\
\hline Typha angustifolia L. & Alt emer Hyd G rhiz & evr-sam (subbor-temp) \\
\hline URTICACEAE & & \\
\hline Urtica dioica L. & a Mes-Alt H scap & evr-sam (bor-temp) \\
\hline Urtica urens L. & a Mes-Meg T scap & evr-sam (bor-temp) \\
\hline VERBENACEAE & & $3,3 \%$ \\
\hline Verbena officinalis L. & a Mes-Meg H scap & kosm (evr-s.afr) \\
\hline VIOLACEAE & & $66,6 \%$ \\
\hline Viola arvensis Murray & v-aut Mi-Mes T scap/a H scap bienn & evr (subbor-merid) \\
\hline Viola tricolor L. & v-a Mes T scap & evr (temp-submerid) \\
\hline
\end{tabular}


According to the literature data on vascular flora of Serbia, the families with the greatest number of species are: Asteraceae, Poaceae, Fabaceae, Caryophyllaceae, Brassicaceae, Lamiaceae, Scrophulariaceae, Apiaceae (Stevanović et al., 1995). In the ruderal flora of Kosovska Mitrovica and its surroundings the largest families coincide with the most numerous families in the flora of entire Serbia (Asteraceae-77 species, Poaceae-49 species, Fabaceae- 46 species, Brassicaceae-36, Lamiaceae- 22 species, Caryophyllaceae-20 species, Apiaceae-16 species, Scrophulariaceae-12 species). The result was anticipated because of the synanthropic character of a great number of representatives of these families.

The most profuse genera found within the taxonomic floral structure in Kosovska Mitrovica area were the following: Vicia (11 sp.), Trifolium (9 sp.), Veronica (8 sp.), Medicago (7 sp.), Bromus and Ranunculus (each with 6 sp.), Centaurea, Crepis, Sonchus, Silene, Euphorbia, Poa, (each with 5 sp.), Cirsium, Rorippa, Geranium, Salvia and Rumex (each with $4 \mathrm{sp}$.). These genera usually include a large number of ruderal and ruderal-segetal plants. The species comprising these genera show the anthropogenic character of diverse ruderal habitats indicating either their nitrification or their xerothermic features, or the presence of intensive treading, mowing, and other anthropogenic influences that make ruderal habitats highly dynamic and instable biotopes (Jovanović, 1994).

\section{General life form spectrum}

The flora of Central European cities is dominated by hemicryptophytic life forms (Sukopp, 1990), while therophitic life forms prevail in South European city flora (Stešević et al., 2014). The ecological analysis in Kosovska Mitrovica showed the hemicryptophitic type of ruderal flora (189 sp. 42,56\% of total) (Fig. 2). The dominant role of hemicryptophytes is potentially even more emphasized by the fact that among the primary annual (one year old) plants (therophytes) there are 25 species that, under specific conditions, alternate as two years old forms. The hemicryptophyte life form is largely described by trunk (stem) shapes (H scap), with 111 species. The domination of hemicryptophytes in the biological spectrum of the ruderal flora of Kosovska Mitrovica corresponds to a dominant presence of these life forms in the whole flora of the Balkans (Turill, 1929). Repeated field researches, 20 years after, showed that the number of hemicryptophytes in the ecological spectrum of life forms had increased by 19 representatives. This may be confirmed by the fact that some surfaces where the floral material was collected have ecologically stabilized.

Therophytes participated less (179 sp., 40,31\% of total), which classified them as second in the biological spectrum of Kosovska Mitrovica ruderal flora. As a rule, the higher the impact of anthropogenic factor in a ruderal habitat, the greater the participation of therophytes on account of one and two years old species, which leads to the biospectrum modification (Jovanović, 1994). The total number of the newly reported or discovered plants that belong to this life form is 16. Other life forms were present with a smaller number of representatives: geophytes (25 sp., 5,63\%), phanerophytes (20 sp., 4,50\%), chamaephytes and scandentophytes with equal number of species (12 sp., 2,70\%), and hydrophytes with 7 sp., $1,57 \%$. 


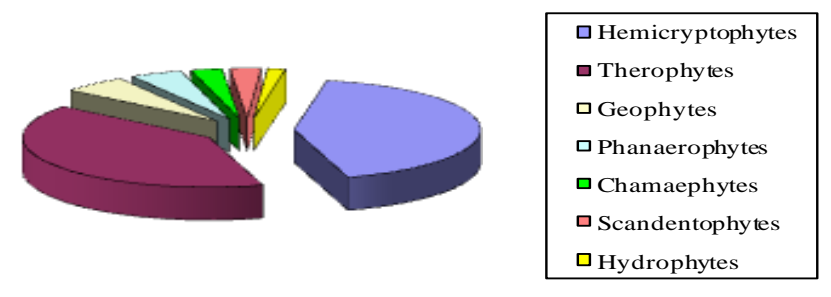

Figure 2. Life form spectrum of ruderal flora of Kosovska Mitrovica (Serbia)

\section{Phytogeographical analysis of ruderal flora}

Ruderal flora, as a specific plant category, is under the anthropogenic impact. It is unsuitable for the analysis whose aim is to determine floral and geographical belonging to certain area (Jovanović, 1985). This could be explained by the fact that ruderal flora encompasses species of vast areas whose diffusion was (directly or indirectly) influenced by man. There is also a fact that the composition and diversity /abundance of ruderal flora and vegetation of some area are proportional to the intensity of anthropogenic influences and may serve as an indicator of environmental quality and urbanization level. A detailed phytogeographic analysis of Kosovska Mitrovica ruderal flora corroborated the presence of 7 basic area types that incorporate 18 areal groups (Fig. 3).

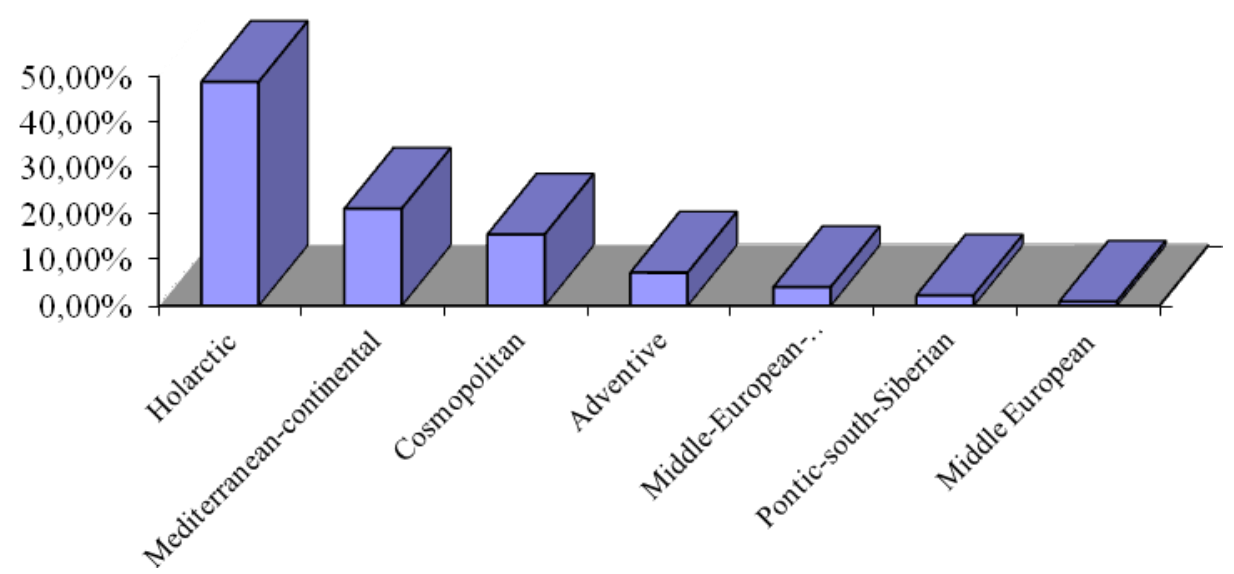

Figure 3. Chorological spectrum, by basic area types, of the entire ruderal flora of Kosovska Mitrovica (Serbia)

Most numerous species were of the Holarctic area type, presented with 217 species or $48,87 \%$, within which the European, Western-Asian and Euroasian group of floristic elements dominated. According to its number, Mediterranean-continental type is 
second, with 94 taxa $(21,17 \%)$ united in 4 different groups of area floral elements. The areal types of Cosmopolitan species are represented by 69 taxa $(15,54 \%)$. The cosmopolits of Euroasian and Mediterranean origin are larger in number compared to the Cosmopolits of circumholarctic and tropical origin.

The presence of adventive plant species is relatively high (32 taxa or 7,21\%). The increased number of adventive and cosmopolitan species shows the instability of urban and suburban habitats, because 19 new taxa have been reported in the past 20 years (from the total of 58 recently recorded) and they all belong to the given area type. The common representatives are: Symphyotrichum novi-belgii (L.) G.L.Nesom, Symphyotrichum salignum (Willd.) G.L.Nesom, Calendula officinalis L., Helianthus tuberosus L., Solidago gigantea Aiton, Phytolacca americana L., Antirrhinum majus L., etc. It could be noted that they are the species that man brings into the urban habitats, mostly as decorative and horticulture plants. Due to their pronounced biological potential they conquer new habitats and anthropogenically spread from urban areas to natural (uncultivated) surroundings, usually after removing the plant remnants in autumn and placing them in landfills /disposal sites. The least reported species in the ruderal flora of Kosovska Mitrovica is Middle-European-Mediterranean areal type (18 species), Ponto-South Siberian (10 species) and Middle European dispersion areal type (4 species).

\section{Ecological analysis of ruderal flora}

The contemporary way of life and urbanization ( demolition and new construction, changes in land use, traffic, etc.) in the town have resulted in fragmentation of habitats or their complete loss. Rapid urbanisation in Kosovska Mitrovica over the last decade has caused a visible decrease in green areas, but also the change of floral composition of the ruderal flora. Following the dynamics of appearance of the found ruderal floras in certain localities, it is possible to ascertain their ecological (phenotypic) plasticity and their adaptability to various conditions that prevail on investigated surfaces. The most widespread species Convolvulus arvensis is the most prevailing species $(93,3 \%)$ in 28 out of 30 researched areas. The given species inhabits sunny and open ruderal habitats where it vies with other species for nutrients and available humidity. It also may decrease habitat biodiversity. It is one of the most serious weeds of agricultural fields in temperate regions of the world.

According to its prevalence degree of $76,6 \%$ and phenotypic plasticity and adaptability, Taraxacum officinale occupies the second place and inhabits the most diverse ruderal habitats, which may be confirmed by the results of the comparative morpho-anatomical research of the species $T$. officinale whose samples originate from trodden and untrodden areas of Belgrade (Stevanović et al., 1988). From the ecoclimatic point of view, the trodden ruderal habitats along with the majority of other types of ruderal habitats, may be defined as xerothermic with a favourable regime but with weak soil aeration and severe water deficiency. Taraxacum officinale has very large leaves with mesomorphic structure on untrodden surfaces. The members of this species on trodden areas show a variety of xeromorphic features. This indicates a significant phenotypic plasticity of the species and their adaptability to the changed environments in different ruderal habitats.

Species that were found in more than $50 \%$ of the sampling sites were: Plantago lanceolata, Capsella bursa-pastoris, Cardaria draba, Lactuca serriola, Lotus corniculatus (prevalence $=66,66 \%$ ), Artemisia vulgaris, Stellaria media, Chenopodium 
album, Melilotus officinalis, Achillea millefolium (prevalence=60\%), Polygonum aviculare, Medicago lupulina, Cynodon dactylon, Anisantha sterilis, Poa nemoralis, Hordeum murinum subsp. leporinum (prevalence=53,3\%). The group of eurivalent species with $50 \%$ of frequency includes: Silene vulgaris, Tragopogon dubius, Trifolium repens, Tripleurospermum inodorum, Erodium cicutarium, Galium aparine, Cichorium intybus and Ballota nigra. The species like Convolvulus arvense, Taraxacum officinale, Polygonum aviculare, Cynodon dactylon are prominent due to their exceptionally pioneering character and the ability to adapt to diverse, often extreme and difficult conditions of ruderal habitats.

Diametrically opposed to the group of highly frequent ruderal species is a number of species with a limited range-stenovalent plants (prevalence $=6,6 \%$ or $3,3 \%$ ), which , in accordance with their ecology, develop only in certain ruderal habitats. The group of stenovalent plants is represented by ruderal higrophile species that appear on nitrified, humid and periodically flooded river banks, such as: Bidens tripartitus, Persicaria maculosa, Persicaria lapathifolia, Mentha aquatica, Mentha longifolia etc. This group may also include species such as: Comandra elegans, Asparagus tenuifolius and they appear only in one investigated area, as the remains of the primary vegetation form. We assume that, as the anthropogenic influence grows and intensifies in the mentioned localities, some of these species will become extinct because the ruderal habitats do not suit them, i.e. they do not provide ecological optimum for their development.

Sukopp (1973) presents data that between 1850 - 1950 some European cities faced the loss of flora by 4-16\%. Klotz (1987) and Landolt (2000) show that the number of species in the cities flora has remained approximately the same in the past 1-2 centuries, but the flora composition has changed by $30-40 \%$ and provided an advantage for the adventive species (Stešević et al., 2014). Our analyses show that the ruderal flora composition in Kosovska Mitrovica area has changed by $13.06 \%$ in the past 20 years. It is also noted that there has not been a reduction in a total number of species. Autochthonous species were replaced by allochthonous-cosmopolitan species, which as more adaptable to homogenous and degraded urban habitats, are beginning to compete with natural species and are gradually ousting them.

The introduced species that might become invasive represent a special menace and may cause destruction of natural ecosystems and changes in their biodiversity. Newly introduced plants most frequently inhabit ruderal habitats that are temporarily or permanently under the anthropogenic influence. The most widely accepted definition of invasive species is the one advocated by the global environmental organisation IUCN (International Union for Conservation of Nature): "An invasive alien species (IAS) is a species that is established outside of its natural past or present distribution, whose introduction and/or spread threaten biological diversity" Convention on Biological Diversity. Out of total number, 38 plant species from the weed ruderal flora of Kosovska Mitrovica and its surroundings, are labelled as invasive (Acer negundo, Ambrosia artemisifolia, Symphyotrichum novi-belgii, Symphyotrichum salignum, Erigeron canadensis, Galinsoga parviflora, Helianthus tuberosus, Solidago gigantea, Xanthium orientale subsp. italicum, Xanthium spinosum, Centaurea solstitialis, Artemisia vulgaris, Cichorium intybus, Amaranthus albus, Amaranthus blitoides, Amaranthus retroflexus, Bifora radians, Armoracia rusticana, Coronopus squamatus, Echinocystis lobata, Chenopodium strictum, Amorpha fruticosa, Medicago sativa, Robinia pseudoacacia, Alceae rosea, Echiochloa crus-galli, Sorghum halepense, Dasypyrum villosum, Phytolacca americana, Polygonum aviculare, Portulaca 
oleraceae, Consolida orientalis, Consolida regalis, Rubus caesius, Veronica persica, Datura stramonium, Lycium barbarum, Urtica dioica). The largest number of their representatives belong to Asteraceae family (12 species), followed by Amaranthaceae, Fabaceae and Poaceae (each with 3 species). The investigations show that there has been an increase of invasive species and they have become more frequent in every investigated locality over the past 20 years.

If we analyse the origin of invasive species within weed ruderal flora of Kosovska Mitrovica and its surroundings, we may come to the conclusion that the species originating from North American continent (19 of them-50\%) prevail in number. These species were mostly introduced as decorative plants (Robinia pseudoacacia, Acer negundo), but they soon "escaped" to the wilderness attacking the natural ecosystems with detrimental effects and became invasive. It is interesting to mention the Ambrosia artemisfolia species unreported in Kosovska Mitrovica and its surroundings until 2012 has been randomly spotted on certain ruderal places in the city core ever since.

Currently, the larger part of the society perceive ruderal urban plants as common weeds and do not appreciate their stability and aesthetic value. Currently the aesthetic and ecological values of such plant communities are indiscernible and underestimated. However, colourful flowering plant communities in cities have a positive influence on human senses (Kazimierska et al., 2009). Except in the case of allergenic plants and their negative influence on human health, it can be generally stated that the flora and vegetation of man-made environments represent a significant, spontaneously developed phytohealing factor, the one which in such polluted conditions produces a whole series of positive effects so they deserve a better treatment (Stešević and Jovanović, 2008).

In an industrial city, such as Kosovska Mitrovica, a spontaneously developed plant cover has undoubtedly many positive effects ( oxygen production, carbon-dioxide assimilation, soil overheating avoidance, soil erosion prevention, esthetic and healthhygienic protection of the unhygienic areas, decoration) so it should be "exploited" and adjusted to fit human needs (which implies prevention of undesirable species spreading, their blooming and fruiting) and organize a monitoring process over an extended period of time to evaluate the changes in habitat conditions, as well as in floristic composition.

\section{Conclusion}

Due to expected and intense anthropogenic activity on ruderal flora in Kosovska Mitrovica and its surroundings, there was a change in overall floral composition by $13,06 \%$, over a period of 20 years (1996-2016). A total of 444 taxa of vascular plants classified into 271 genera and 58 families were found in various types of ruderal habitats; with 58 new taxa reported in recent field explorations. An analysis of life forms in the ruderal flora of Kosovska Mitrovica showed that after a period of 20 years, the investigated area still has hemicryptophyto-therophyte character, with domination of hemicryptophytes. Phytogeographic analysis revealed the presence of 7 basic area types that incorporate 18 areal groups. The most numerous species were of the Holarctic area type, presented with 217 species or $48,87 \%$. The increased number of adventive and cosmopolitan species shows the instability of urban and suburban habitats, because 19 new taxa have been reported in the past 20 years (from the total of 58 recently recorded) and they all belong to the given area type. The most prevailing species are 
Convolvulus arvense and Taraxacum officinale. Out of total number of recorded species, 38 are labelled as invasive.

All botanical studies should definitely include monitoring biodiversity of urban flora and new short term researches. It is the only method to identify potential threats to autochtonous flora and to enable a timely anthropogenous action to curb spreading of invasive, potentially allergenic species as well as aggressive, competitive species that might have negative effects on human population and urban habitats.

\section{REFERENCES}

[1] Celesti-Grapow, L., Pyšek, P., Jarošǐk, V., Blasi, C. (2006): Determinants of native and alien species richness in the urban flora of Rome. - Diversity and Distributions 12: 490501.

[2] Ellenberg, H., Mueller-Dambois, D. (1967): A key to Raunkiaer plant life forms with revised subdivision. - BerGeobotInst ETH, Zürich, Stifg Rübel 37: 56-73.

[3] International Union for Conservation of Nature: http://www.iucn.org/about/union/secretariat/offices/europe/european_union/key_issues/in vasive_alien_species/

[4] Jarić, S., Mitrović, M., Vrbničanin, S., Karadžić, B., Đurđević, L., Kostić, O., Mačukanović-Jocić, M, Gajić, G., Pavlović, P. (2011): A contribution to studies of the ruderal vegetation of Southern Srem, Serbia. - Arch. Biol. Sci., Belgrade 63(4): 11811197.

[5] Josifović, M. (ed.) (1970-1977): Flora SR Srbije, 1-9. - SANU, Beograd. (In Serbian).

[6] Jovanović, S. (1985): Analysis of ruderal flora northeastern part of Belgrade. Biosistematika, Acta Biologica Iugoslavica, Serija G, Beograd 11(1): 17-30. (In Serbian).

[7] Jovanović, S.(1994): Ecological Study of Ruderal Flora and Vegetation in the City of Belgrade. - Biološki fakulet Univerziteta u Beogradu. 222. (In Serbian).

[8] Jovanović, S.(1997): Mediterranean floristic elements in the ruderal flora of Belgrade (Yugoslavia). - Bocconea 5(2): 439-443.

[9] Jovanović, S., Mitrović, V.(1998): Ruderal Flora of Loznica-Ecological and Phytogeographic characteristics. - Acta herbologica 7(1-2): 37-62.

[10] Kazimierska, N., Szymura, M., Wolski, K.(2009): Aesthetic aspects of plant communities of ruderal urban sities in Szczecin. - Biodiv. Res. Conserv. 13: 43-48.

[11] Marković, Lj. (1964): Phytocenological research of ruderal vegetation in Croatia, PhD thesis, University in Zagreb, Faculty of Natural Science, Zagreb, Yugoslavia (In Croatian).

[12] Marković, Lj. (1978): Ubersicht der Ruderalpflanzen-gesellschaften in Jugoslawien.Acta Bot. Slov. Acad. Sci. Slovacae, Bratislava. Ser.A, 3: 305-309.

[13] Marković, Lj. (1984): Ruderal vegetation of Gorski Kotar. - Acta Bot. Croat. 43: 257272.

[14] Matvejeva, J. (1982): Ruderal vegetation of SR Macedonia. - MANU, Skopje, (In Macedonian).

[15] Neto, M., Otsubo, H., Scabora, M., Maltoni, K., Cassiolato, A.(2015): A Floristic Survey, Origin and Mycorrhization of Ruderal Plants in Remaining Cerrado Areas Publishing Agreement. - Journal of Agriculture and Life Science 2( 2): 38-50.

[16] Pavlović-Muratspahić, D., Stanković, M., Branković, S. (2010): Taxonomical analysis of ruderal flora (sensu stricto) in area of the city of Kragujevac. - Kragujevac Journal of Science 32: 101-108.

[17] Prodanović, D., Krivošej, Z., Jovanović, S. (2008): Ecological and phytogeographical characteristics of ruderal flora in Kosovska Mitrovica and its surroundings. - Natura Montenegrina 7 (3): 307-327. 
[18] Pyšek, P. (1998): Alien and native species in Central European urban floras: a quantitative comparison. - Journal of Biogeography 25: 155-163.

[19] Pyšek, P., Lambdon, Ph., Arianoutsou, M., Kühn, I., Pino, J., Winter, M., (2009): Alien Vascular Plants of Europe. - In: DAISIE, Handbook of Alien species in Europe. Springer: 43-61.

[20] Pyšek, P., Jarošik, V., Pergl, J., Randall, R., Chytry, M., Kühn, I., Tichý, L., Danihelka, J., Chrtek, J., Sádlo, J. (2009): The global invasion success of Central European plants is related to distribution characteristics in their native range and specias traits. - Diversity and Distributions 15: 891-903.

[21] Sarić, M., Diklić, N. (ed.) (1989): Flora SR Srbije 10 (2), SANU, Beograd. (In Serbian).

[22] Slavnić, Ž. (1951): Review of nitrophilous vegetation in Vojvodina. - Proceeding of Matica srpska, series for natural sciences 1: 84-169.

[23] Slavnić, Ž. (1960): About immigration, expansion and domestication of some alien plants in Bosnia and Herzegovina. - Yearbook of the Biological Institute University in Sarajevo 1-2: 117-146 (In Serbian).

[24] Slavnić Ž.(1961): On some adventives species in Vojvodini. - Proceeding of Matica Srpska, series for natural sciences 20: 5-10.

[25] Stanković-Kalezić, R. (2007): Sinecology and floristic study of ruderal vegetation at the area of Pančevački rit. $\mathrm{PhD}$ Thesis, University in Belgrade, Faculty of Agriculture,Belgrade, Serbia (In Serbian).

[26] Stanković-Kalezić, R., Radivojević, Lj., Jovanović, V., Janjić, V., Šantrić, Lj. (2008): Adventive species Asclepias syriaca L. at the area of Pančevački rita. - Acta herbologica, 17(1): 95-103.(In Serbian).

[27] Stanković-Kalezić, R., Jovanović, S., Janjić, V., Radivojević, Lj. (2009): Association Arctio-Artemisietum vulgaris (Tx.1942) Oberd. et al. 1967. the most common ruderal association at the area of Pančevački rit. - Pesticidi i fitomedicina 24(2): 113-121.(In Serbian).

[28] Stešević, D., Jovanović, S. (2008): Flora of the city of Podgorica, Montenegro (taxonomic analysis). - Arch. Biol. Sci., Belgrade 60(2): 245-253.

[29] Stešević, D., Jovanović, S., Šćepanović, S.(2009): Flora of the city of Podgorica, Montenegro-chorologic structure and comparisom with the floras of Rome, Patras, and Salonika. - Arch. Biol. Sci., Belgrade 61(2): 307-315.

[30] Stešević, D., Caković, D., Jovanović, S. (2014): The Urban Flora of Podgorica (Montenegro, SE Europe): Annotated Checklist, Distribution Atlas, Habitats And LifeForms,Taxonomic, Phytogeographical And Ecological Analysis. - Ecologica Montenegrina, Supplementum 1: 1-171.

[31] Stevanović, B., Jovanović, S., Šošić, Lj.(1988): Ecological characteristics of ruderal vegetation. I-morpho-anatomical analysis of ruderal plants from trampled and untrodden ruderal areas. - Gazette of Institute of Botany and botanical garden University of Belgrade 22: 117-130. (In Serbian).

[32] Stevanović, V. (1992): The classification of life forms in the flora of Serbia. - In: Sarić, M. (ed) Flora Srbije 1 (2.ed.) - SANU, Beograd: 39-46. (In Serbian).

[33] Stevanović, V. (1992a): Floristic division of the territory of Serbia with an overview of higher horions and appropriate floral elements. - In Sarić,M. (ed.) Flora Srbije 1 (2.ed.), SANU, Beograd: 49-65.(In Serbian).

[34] Stevanović, V., Jovanović, S., Lakušić, D., Niketić, M. (1995): Diversity of vascular plants of Yugoslavia, with review of internationally significant species. - In: Stevanović, V., Vasić, V. (ed.) Biodiversity of Yugoslavia with an overview of species of global importance. Ecolibri: Biološki fakultet, Belgrade: 183-217.(In Serbian).

[35] Sukopp, H.(1990): Stadtökologie. Das Beispiel Berlin. - Dietrich ReimerVerlag, Berlin. 455.

[36] Sukopp, H. (2002): On the early history of urban ecology in Europe. - Preslia, Praha 74: 373-393. 
[37] Šajinović, B. (1968): Ecologically -phytocoenological analysis of ruderal vegetation in Novi Sad surrounding. MsC Thesis, Faculty of Natural Science, University of Belgrade.Belgrade, Serbia (In Serbian).

[38] The Euro+Med Plantbase (2006-) : The information resource for Euro-Mediterranean plant diversity. Available at: http://ww2. bgbm.org/EuroPlusMed

[39] The Plant List (2013): Version 1.1. Published on the Internet; http://www. theplantlist.org/ (accesses $1^{\text {st }}$ January).

[40] Turrill, WB. (1929): The Plant Life of the Balkan Peninsula. A Phytogeographical Study. - Clarendon. Oxford.

[41] Tutin, TG. (ed.) (1964-1980): Flora Europaea 1-5. - Cambridge University Press, London.

[42] Williams, N., Schwartz, M., Vesk, P., McCarthy, M., Hahs, A., Clemants, S., Corlett, R., Duncan, R., Norton, B., Thompson, K., McDonell, M. (2008): A conceptual framework for predicting the effects of urban environment on floras. - Journal $f$ Ecology. British Ecological Society: 1-6. 